\title{
Fibered orbifolds and crystallographic groups
}

\author{
JOHN G RATCLIFFE \\ STEVEN T TSCHANTZ
}

\begin{abstract}
In this paper, we prove that a normal subgroup $\mathrm{N}$ of an $n$-dimensional crystallographic group $\Gamma$ determines a geometric fibered orbifold structure on the flat orbifold $E^{n} / \Gamma$, and conversely every geometric fibered orbifold structure on $E^{n} / \Gamma$ is determined by a normal subgroup $\mathrm{N}$ of $\Gamma$. In particular, we prove that $E^{n} / \Gamma$ is a fiber bundle, with totally geodesic fibers, over a $\beta_{1}$-dimensional torus, where $\beta_{1}$ is the first Betti number of $\Gamma$.
\end{abstract}

20H15; 55R65, 57M50, 57S30

\section{Introduction}

Let $E^{n}$ be Euclidean $n$-space. A map $\phi: E^{n} \rightarrow E^{n}$ is an isometry of $E^{n}$ if and only if there is an $a \in E^{n}$ and an $A \in \mathrm{O}(n)$ such that $\phi(x)=a+A x$ for each $x$ in $E^{n}$. We shall write $\phi=a+A$. In particular, every translation $\tau=a+I$ is an isometry of $E^{n}$.

A flat $n$-orbifold is a $\left(E^{n}, \operatorname{Isom}\left(E^{n}\right)\right)$-orbifold as defined in Section 13.2 of Ratcliffe [14]. A connected flat $n$-orbifold has a natural inner metric space structure. If $\Gamma$ is a discrete group of isometries of $E^{n}$, then its orbit space $E^{n} / \Gamma=\left\{\Gamma x: x \in E^{n}\right\}$ is a connected, complete, flat $n$-orbifold, and conversely if $M$ is a connected, complete, flat $n$-orbifold, then there is a discrete group $\Gamma$ of isometries of $E^{n}$ such that $M$ is isometric to $E^{n} / \Gamma$ by Theorem 13.3.10 of [14].

Definition A flat $n$-orbifold $M$ geometrically fibers over a flat $m$-orbifold $B$, with generic fiber a flat $(n-m)$-orbifold $F$, if there is a surjective map $\eta: M \rightarrow B$, called the fibration projection, such that for each point $y$ of $B$, there is an open metric ball $B(y, r)$ of radius $r>0$ centered at $y$ in $B$ such that $\eta$ is isometrically equivalent on $\eta^{-1}(B(y, r))$ to the natural projection $\left(F \times B_{y}\right) / G_{y} \rightarrow B_{y} / G_{y}$, where $G_{y}$ is a finite group acting diagonally on $F \times B_{y}$, isometrically on $F$ and effectively and orthogonally on an open metric ball $B_{y}$ in $E^{m}$ of radius $r$. This implies that the fiber $\eta^{-1}(y)$ is isometric to $F / G_{y}$. The fiber $\eta^{-1}(y)$ is said to be generic if $G_{y}=\{1\}$ or singular if $G_{y}$ is nontrivial. 
An $n$-dimensional crystallographic group (space group) is a discrete group of isometries $\Gamma$ of $E^{n}$ such that $E^{n} / \Gamma$ is compact. We prove that if $\mathrm{N}$ is a normal subgroup of an $n$-dimensional space group $\Gamma$, then the flat orbifold $E^{n} / \Gamma$ geometrically fibers over a flat orbifold, with generic fiber a connected flat orbifold, naturally induced by $\mathrm{N}$. Conversely, we prove that if $E^{n} / \Gamma$ geometrically fibers over a flat orbifold $B$ with generic fiber a connected flat orbifold $F$, then this fibration is equivalent to a geometric fibration induced by a normal subgroup $\mathrm{N}$ of $\Gamma$.

An $m$-dimensional torus or $m$-torus is a topological space homeomorphic to the cartesian product of $S^{1}$ with itself $m$ times. Here a 0 -torus is defined to be a point. We prove that if $\Gamma$ is an $n$-dimensional space group with first Betti number $\beta_{1}$, then the flat orbifold $E^{n} / \Gamma$ is a fiber bundle over a $\beta_{1}$-torus with totally geodesic fibers.

Let $\mathrm{N}$ be a normal subgroup of a space group $\Gamma$. We prove that $\Gamma / \mathrm{N}$ is a space group if and only if $\mathrm{N}$ is maximal in the commensurability class of normal subgroups of $\Gamma$ that contain $N$. Suppose $\Gamma / N$ is a space group. We study the relationship between the exact sequence $1 \rightarrow \mathrm{N} \rightarrow \Gamma \rightarrow \Gamma / \mathrm{N} \rightarrow 1$ splitting and the corresponding fibration projection having an affine section. If $\mathrm{N}$ is torsion-free, we prove that the exact sequence splits if and only if the fibration projection has an affine section. If the generic fiber $F=\operatorname{Span}(\mathrm{N}) / \mathrm{N}$ has an ordinary point that is fixed by every isometry of $F$, we prove that the exact sequence always splits.

Finally, we illustrate the theory by describing all the geometric fibrations of the orbit spaces of all 2- and 3-dimensional crystallographic groups building on the work of Conway et al [7].

\section{Normal subgroups of crystallographic groups}

The fundamental theorem of crystallographic groups is the following theorem.

\section{Theorem 1 (Bieberbach's Theorems)}

(1) If $\Gamma$ is an $n$-dimensional space group, then the subgroup $\mathrm{T}$ of all translations in $\Gamma$ is a free abelian normal subgroup of rank $n$ and of finite index in $\Gamma$ such that $\{a: a+I \in \mathrm{T}\}$ spans $E^{n}$.

(2) Every isomorphism between $n$-dimensional space groups $\Gamma_{1}$ and $\Gamma_{2}$ is equal to conjugation by an affine homeomorphism of $E^{n}$.

(3) There are only finitely many isomorphism classes of $n$-dimensional space groups for each $n$. 
In dimensions $0,1, \ldots, 6$, there are $1,2,17,219,4783,222018,28927922$ isomorphism classes of space groups, respectively. For the classification of these groups; see Brown et al [3] and Plesken and Schulz [13].

Let $\Gamma$ be an $n$-dimensional space group. Define $\eta: \Gamma \rightarrow \mathrm{O}(n)$ by $\eta(a+A)=A$. Then $\eta$ is a homomorphism whose kernel is the group of translations in $\Gamma$. The image $\Pi$ of $\eta$ is a finite group by Part (1) of Theorem 1 called the point group of $\Gamma$. Let $\mathrm{N}$ be a normal subgroup of $\Gamma$. Define

$$
\operatorname{Span}(\mathrm{N})=\operatorname{Span}\left\{a \in E^{n}: a+I \in \mathrm{N}\right\} .
$$

Note that $\operatorname{Span}(\mathrm{N})$ is a vector subspace $V$ of $E^{n}$. Let $V^{\perp}$ denote the orthogonal complement of $V$ in $E^{n}$. The following theorem strengthens Theorem 17 of Farkas [9].

Theorem 2 Let $\mathrm{N}$ be a normal subgroup of an $n$-dimensional space group $\Gamma$, and let $V=\operatorname{Span}(\mathrm{N})$.

(1) If $b+B \in \Gamma$, then $B V=V$.

(2) If $a+A \in \mathrm{N}$, then $a \in V$ and $V^{\perp} \subseteq \operatorname{Fix}(A)$.

(3) The group $\mathrm{N}$ acts effectively on each coset $V+x$ of $V$ in $E^{n}$ as a space group of isometries of $V+x$.

Proof (1) Let $a+I \in \mathrm{N}$ and let $b+B \in \Gamma$, then $(b+B)(a+I)(b+B)^{-1}=$ $B a+I \in \mathrm{N}$. Hence $B$ leaves $V$ invariant.

(2) The coset space $E^{n} / V$ is a Euclidean space where the distance between cosets is the orthogonal distance in $E^{n}$. The quotient map from $E^{n}$ to $E^{n} / V$ maps $V^{\perp}$ isometrically onto $E^{n} / V$. The group $\Gamma$ acts isometrically on $E^{n} / V$ by $(b+B)(V+x)=$ $V+b+B x$.

Let $\mathrm{T}=\{a+I \in \Gamma\}$. Then $\mathrm{N} / \mathrm{N} \cap \mathrm{T} \cong \mathrm{NT} / \mathrm{T} \subseteq \Gamma / \mathrm{T}$, and so $\mathrm{N} / \mathrm{N} \cap \mathrm{T}$ is a finite group. The group $\mathrm{N} \cap \mathrm{T}$ acts trivially on $E^{n} / V$, and so $\mathrm{N} / \mathrm{N} \cap \mathrm{T}$ acts isometrically on $E^{n} / V$. Therefore $\mathrm{N} / \mathrm{N} \cap \mathrm{T}$ fixes a point $V+x$ of $E^{n} / V$. This implies that the group $\mathrm{N}$ leaves the coset $V+x$ invariant. Let $\mathrm{N}^{\prime}=(-x+I) \mathrm{N}(x+I)$. Then $\mathrm{N}^{\prime}$ leaves $V$ invariant. Let $a+A \in N$. Then $(-x+I)(a+A)(x+I)=a+A x-x+A$. Let $a^{\prime}=a+(A-I) x$. Then $a^{\prime}+A \in \mathrm{N}^{\prime}$. As $a^{\prime}+A$ leaves $V$ invariant, $a^{\prime} \in V$.

Let $b+I \in \Gamma$. Then $b+I \in(-x+I) \Gamma(x+I)$, since $b+I$ and $x+I$ commute. Let $a+A \in \mathrm{N}$. Then $(-b+I)\left(a^{\prime}+A\right)(b+I)=a^{\prime}+(A-I) b+A$ is in $\mathrm{N}^{\prime}$. Hence $(A-I) b \in V$. Now $\{b: b+I \in \Gamma\}$ spans $E^{n}$ by Theorem 1 . Let $W=(\operatorname{Fix}(A))^{\perp}$. Then $A-I$ maps $W$ isomorphically onto $W$, and so $A-I$ maps $E^{n}$ onto $W$. Hence $\{(A-I) b: b+I \in \Gamma\}$ spans $W$. Therefore $W \subseteq V$. Hence $V^{\perp} \subseteq W^{\perp}=\operatorname{Fix}(A)$. 
Let $a+A \in \mathrm{N}$. Write $a=b+c$ with $b \in V$ and $c \in V^{\perp}$. Let $r$ be the order of $A$. Then $r$ is finite and

$$
\begin{aligned}
(a+A)^{r} & =a+A a+\cdots+A^{r-1} a+I \\
& =b+A b+\cdots+A^{r-1} b+r c+I=d+I .
\end{aligned}
$$

As $b+A b+\cdots+A^{r-1} b, d \in V$, we have $r c=0$, and so $c=0$. Hence $a \in V$.

(3) If $a+A \in \mathrm{N}$, then $a \in V$ and $A V=V$, and so $(a+A) V=V$. Hence the group $\mathrm{N}$ leaves $V$ invariant. As $V^{\perp} \subseteq \operatorname{Fix}(A)$ for each $a+A \in \mathrm{N}$, we deduce that $\mathrm{N}$ acts effectively on $V+x$ for each $x \in V^{\perp}$.

Let $a_{1}+I, \ldots, a_{k}+I$ be translations in $\mathrm{N}$ such that $\left\{a_{1}, \ldots, a_{k}\right\}$ is a basis for $V$. Then $(V+x) /\left\langle a_{1}+I, \ldots, a_{k}+I\right\rangle$ is a $k$-torus for each $x \in V^{\perp}$. Hence $(V+x) / \mathrm{N}$ is compact for each $x \in V^{\perp}$. Therefore $\mathrm{N}$ acts effectively as a space group of isometries of $V+x$ for each $x \in V^{\perp}$.

Let $\mathrm{N}$ be a normal subgroup of an $n$-dimensional space group $\Gamma$, and let $V=\operatorname{Span}(\mathrm{N})$. The group $\mathrm{N}$ acts trivially on $E^{n} / V$ by Theorem 2 , and so $\Gamma / \mathrm{N}$ acts isometrically on $E^{n} / V$ by

$$
(\mathrm{N}(b+B))(V+x)=\mathrm{N}((b+B)(V+x))=V+b+B x .
$$

Observe that $\mathrm{N}(b+B)$ fixes $V$ if and only if $b \in V$. Hence the kernel of the corresponding homomorphism from $\Gamma / \mathrm{N}$ to $\operatorname{Isom}\left(E^{n} / V\right)$ is $\overline{\mathrm{N}} / \mathrm{N}$ where

$$
\overline{\mathrm{N}}=\left\{b+B \in \Gamma: b \in V \text { and } V^{\perp} \subseteq \operatorname{Fix}(B)\right\} .
$$

Hence $\Gamma / \mathrm{N}$ acts effectively on $E^{n} / V$ if and only if $\overline{\mathrm{N}}=\mathrm{N}$. Now as $\overline{\mathrm{N}} / \mathrm{N}$ is a normal subgroup of $\Gamma / \mathrm{N}$, we have that $\overline{\mathrm{N}}$ is a normal subgroup of $\Gamma$. As $V=\operatorname{Span}(\overline{\mathrm{N}})$, we have that $\overline{\mathrm{N}}$ acts effectively as a space group of isometries of $V$ by Part (3) of Theorem 2. Therefore $\mathrm{N}$ has finite index in $\overline{\mathrm{N}}$.

The group $\mathrm{N}$ may be a proper subgroup of $\overline{\mathrm{N}}$. For example, if $\mathrm{N}$ is the group of translations of $\Gamma$, then $\overline{\mathrm{N}}=\Gamma$.

Two subgroups $\mathrm{H}_{1}$ and $\mathrm{H}_{2}$ be subgroups of a group $\Gamma$ are said to be commensurable if $\mathrm{H}_{1} \cap \mathrm{H}_{2}$ has finite index in both $\mathrm{H}_{1}$ and $\mathrm{H}_{2}$. Commensurability is an equivalence relation on the set of subgroups of $\Gamma$.

Theorem 3 Let $\mathrm{N}_{1}$ and $\mathrm{N}_{2}$ be normal subgroups of a space group $\Gamma$. Then $\overline{\mathrm{N}_{1}}=\overline{\mathrm{N}_{2}}$ if and only if $\mathrm{N}_{1}$ and $\mathrm{N}_{2}$ are commensurable. 
Proof Suppose $\mathrm{N}_{1}$ and $\mathrm{N}_{2}$ are commensurable. Let $\mathrm{T}=\{a+I \in \Gamma\}$. Now $\mathrm{N}_{1} \cap \mathrm{N}_{2} \cap \mathrm{T}$ has finite index in $\mathrm{N}_{1} \cap \mathrm{N}_{2}$ and $\mathrm{N}_{1} \cap \mathrm{N}_{2}$ has finite index in $\mathrm{N}_{i}$ for $i=1,2$. Hence $\mathrm{N}_{1} \cap \mathrm{N}_{2} \cap \mathrm{T}$ has finite index in $\mathrm{N}_{i} \cap \mathrm{T}$ for $i=1,2$. Therefore $\operatorname{Span}\left(\mathrm{N}_{1} \cap \mathrm{N}_{2}\right)=\operatorname{Span}\left(\mathrm{N}_{i}\right)$ for $i=1,2$. Hence $\overline{\mathrm{N}_{1}}=\overline{\mathrm{N}_{2}}$.

Conversely, suppose $\overline{\mathrm{N}_{1}}=\overline{\mathrm{N}_{2}}$. Now $\mathrm{N}_{i}$ has finite index in $\overline{\mathrm{N}_{i}}$ for $i=1,2$. As $\mathrm{N}_{1} \mathrm{~N}_{2} \subseteq \overline{\mathrm{N}_{1}}=\overline{\mathrm{N}_{2}}$, we have that $\mathrm{N}_{i}$ has finite index in $\mathrm{N}_{1} \mathrm{~N}_{2}$ for $i=1,2$. Now $\mathrm{N}_{1} / \mathrm{N}_{1} \cap \mathrm{N}_{2} \cong \mathrm{N}_{1} \mathrm{~N}_{2} / \mathrm{N}_{2}$ and $\mathrm{N}_{2} / \mathrm{N}_{1} \cap \mathrm{N}_{2} \cong \mathrm{N}_{1} \mathrm{~N}_{2} / \mathrm{N}_{1}$. Hence $\mathrm{N}_{1} \cap \mathrm{N}_{2}$ has finite index in both $\mathrm{N}_{1}$ and $\mathrm{N}_{2}$. Therefore $\mathrm{N}_{1}$ and $\mathrm{N}_{2}$ are commensurable.

Corollary 1 If $\mathrm{N}$ is a normal subgroup of a space group $\Gamma$, then $\overline{\overline{\mathrm{N}}}=\overline{\mathrm{N}}$ and $\overline{\mathrm{N}}$ is the unique maximal element of the commensurability class of normal subgroups of $\Gamma$ that contains $\mathrm{N}$.

Corollary 2 If $\psi: \Gamma \rightarrow \Gamma^{\prime}$ is an isomorphism of space groups, and $\mathrm{N}$ is a normal subgroup of $\Gamma$, then $\overline{\psi(\mathrm{N})}=\psi(\overline{\mathrm{N}})$.

\section{Geometric flat orbifold fibrations}

We say that the normal subgroup $\mathrm{N}$ of a space group $\Gamma$ is a complete if $\overline{\mathrm{N}}=\mathrm{N}$. If $\mathrm{N}$ is a normal subgroup of $\Gamma$, then $\bar{N}$ is a complete normal subgroup of $\Gamma$ by Corollary 1 called the completion of $\mathrm{N}$ in $\Gamma$.

Lemma 1 Let $\mathrm{N}$ be a complete normal subgroup of an $n$-dimensional space group $\Gamma$, and let $V=\operatorname{Span}(\mathrm{N})$. Then $\Gamma / \mathrm{N}$ acts effectively as a space group of isometries of $E^{n} / V$.

Proof From the above discussion, we know that $\Gamma / \mathrm{N}$ acts effectively as a group of isometries of $E^{n} / V$. As $\mathrm{NT} / \mathrm{N}$ is of finite index in $\Gamma / \mathrm{N}$, it suffices to show that $\mathrm{NT} / \mathrm{N}$ acts as a space group of isometries of $E^{n} / V$. Now $\mathrm{NT} / \mathrm{N} \cong \mathrm{T} / \mathrm{N} \cap \mathrm{T}$. We claim that the group $\mathrm{T} / \mathrm{N} \cap \mathrm{T}$ is torsion-free. Let $\tau=a+I \in \mathrm{T}$ and suppose that $\tau^{r} \in \mathrm{N} \cap \mathrm{T}$ for some integer $r>0$. Then $r a+I \in \mathrm{N} \cap \mathrm{T}$. Hence $r a \in V$, and so $a \in V$. Therefore $\tau \in \mathrm{N} \cap \mathrm{T}$, since $\mathrm{N}$ is complete. Thus $\mathrm{T} / \mathrm{N} \cap \mathrm{T}$ is torsion-free. Hence $\mathrm{T}$ has a set of generators $\left\{b_{1}+I, \ldots, b_{n}+I\right\}$ such that $b_{1}+I, \ldots, b_{k}+I$ generate $\mathrm{N} \cap \mathrm{T}$. Then $\mathrm{N}\left(b_{k+1}+I\right), \ldots, \mathrm{N}\left(b_{n}+I\right)$ generate NT/N. As $\left\{b_{k+1}, \ldots, b_{n}\right\}$ projects to a basis of $E^{n} / V$, we have that NT/N acts as a space group of translations of $E^{n} / V$ by Theorems 5.2.4 and 5.3.2 of Ratcliffe [14].

Theorem 4 Let $\mathrm{N}$ be a complete normal subgroup of an $n$-dimensional space group $\Gamma$, and let $V=\operatorname{Span}(\mathrm{N})$. Then the flat orbifold $E^{n} / \Gamma$ geometrically fibers over the flat orbifold $\left(E^{n} / V\right) /(\Gamma / \mathrm{N})$ with generic fiber the flat orbifold $V / \mathrm{N}$. 
Proof Let $\eta: E^{n} / \Gamma \rightarrow\left(E^{n} / V\right) /(\Gamma / \mathrm{N})$ be the map defined by

$$
\eta(\Gamma x)=(\Gamma / \mathrm{N})(V+x)=\Gamma(V+x) .
$$

Then the diagram

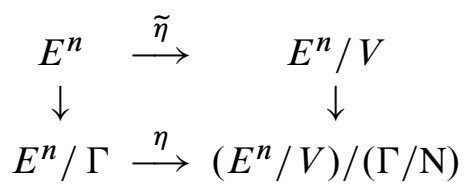

commutes, where $\tilde{\eta}$ and the vertical maps are the quotient maps. Hence $\eta$ is a continuous surjection, and so $\left(E^{n} / V\right) /(\Gamma / \mathrm{N})$ is a compact flat $m$-orbifold with $m=\operatorname{dim}\left(E^{n} / V\right)$.

Let $x \in V^{\perp}$, and let

$$
\Gamma_{V+x}=\{\gamma \in \Gamma: \gamma(V+x)=V+x\} .
$$

Then the stabilizer of $V+x$ in $\Gamma / \mathrm{N}$ is $G_{x}=\Gamma_{V+x} / \mathrm{N}$. By Lemma 1 , the group $G_{x}$ is finite. Let $r$ be half the distance from $V+x$ to the set of remaining points of the orbit $(\Gamma / \mathrm{N})(V+x)$ in $E^{n} / V$. By Lemma 1 , we have that $r>0$. Let $B_{x}$ be the open ball of radius $r$ centered at $V+x$ in $E^{n} / V$. The quotient map from $E^{n} / V$ to $\left(E^{n} / V\right) /(\Gamma / \mathrm{N})$ maps $B_{x}$ onto $(\Gamma / \mathrm{N}) B_{x} /(\Gamma / \mathrm{N})$ which is isometric to $B_{x} / G_{x}$ with $G_{x}$ acting effectively and orthogonally on $B_{x}$.

Let $U$ be the $r$-neighborhood of $V+x$ in $E^{n}$. Then we have

$$
\eta^{-1}\left((\Gamma / \mathrm{N}) B_{x} /(\Gamma / \mathrm{N})\right)=\Gamma U / \Gamma .
$$

Now $\Gamma U / \Gamma$ is isometric to $U / \Gamma_{V+x}$. Moreover we have

$$
U / \Gamma_{V+x}=(U / \mathrm{N}) /\left(\Gamma_{V+x} / \mathrm{N}\right)=(U / \mathrm{N}) / G_{x} .
$$

The group $\mathrm{N}$ acts trivially on $E^{n} / V$. Hence $U / \mathrm{N}$ is isometric to $((V+x) / \mathrm{N}) \times B_{x}$. Let $F=V / \mathrm{N}$. Then $F$ is a compact flat $(n-m)$-orbifold. Now $F$ is isometric to $(V+x) / \mathrm{N}$, since $\mathrm{N}$ acts trivially on $E^{n} / V$. The finite group $G_{x}$ acts diagonally on $((V+x) / \mathrm{N}) \times B_{x}$, isometrically on $(V+x) / \mathrm{N}$ and effectively and orthogonally on $B_{x}$. We have a commutative diagram

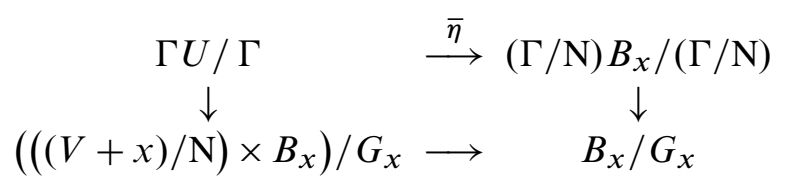

where the vertical maps are isometries, $\bar{\eta}$ is the restriction of $\eta$, and the bottom map is the natural projection. Thus $E^{n} / \Gamma$ geometrically fibers over the flat $m$-orbifold $\left(E^{n} / V\right) /(\Gamma / \mathrm{N})$ with generic fiber the flat $(n-m)$-orbifold $F=V / \mathrm{N}$. 
Let $\mathrm{N}$ be a normal subgroup of an $n$-dimensional space group $\Gamma$. We call the map $\eta: E^{n} / \Gamma \rightarrow\left(E^{n} / V\right) /(\Gamma / \overline{\mathrm{N}})$ defined in the proof of Theorem 4 , the fibration projection determined by $\mathrm{N}$. Let $\mathrm{T}=\{a+I \in \Gamma\}$. By Theorem 4, the fibration projection $\eta$ determined by $\mathrm{N}$ is an injective Seifert fibering, with typical fiber $V /(\mathrm{N} \cap \mathrm{T})$, in the sense of Lee and Raymond [11].

Theorem 5 Let $\mathrm{N}$ be a normal subgroup of a space group $\Gamma$. Then the following are equivalent:

(1) The quotient group $\Gamma / \mathrm{N}$ is a space group.

(2) The quotient group $\Gamma / \mathrm{N}$ has no nontrivial finite normal subgroups.

(3) The normal subgroup $\mathrm{N}$ of $\Gamma$ is complete.

Proof By Theorem 2 every normal subgroup of a space group is a space group. Hence a space group has no nontrivial finite normal subgroups. Therefore (1) implies (2). As $\overline{\mathrm{N}} / \mathrm{N}$ is a finite normal subgroup of $\Gamma / N$, we have that (2) implies (3). Finally (3) implies (1) by Lemma 1.

If $\Gamma$ is a group, let $Z(\Gamma)$ be the center of $\Gamma$. If $\Gamma$ is a finitely generated group, let $\beta_{1}$ be the first Betti number of $\Gamma$. Here $\Gamma /[\Gamma, \Gamma] \cong G \oplus \mathbb{Z}^{\beta_{1}}$ with $G$ a finite abelian group. For the abelianizations of all the $n$-dimensional space groups for $n=1,2,3$, see our paper [15]. The next theorem strengthens Theorem 6 of Farkas [9].

Theorem 6 If $\Gamma$ is a space group, then every element of $Z(\Gamma)$ is a translation, the rank of $Z(\Gamma)$ is $\beta_{1}$, and $Z(\Gamma)$ is a complete normal subgroup of $\Gamma$.

Proof The translations in $\Gamma$ are characterized as the elements of $\Gamma$ with only finitely many conjugates. Hence $Z(\Gamma)$ is a subgroup of the group $\mathrm{T}$ of translations of $\Gamma$.

Let $\Pi$ be the point group of $\Gamma$. If $a+I \in \mathrm{T}$ and $b+B \in \Gamma$, then

$$
(b+B)(a+I)(b+B)^{-1}=B a+I .
$$

Hence conjugation in $\Gamma$ induces an action of $\Pi$ on $T$. The group extension

$$
1 \rightarrow \mathrm{T} \longrightarrow \Gamma \longrightarrow \Pi \rightarrow 1
$$

determines an exact sequence of cohomology groups

$$
H^{1}(\Pi, \mathbb{Z}) \rightarrow H^{1}(\Gamma, \mathbb{Z}) \rightarrow H^{1}(\mathrm{~T}, \mathbb{Z})^{\Pi} \rightarrow H^{2}(\Pi, \mathbb{Z}) .
$$

Here $\mathrm{T}, \Gamma$ and $\Pi$ act trivially on $\mathbb{Z}$ and $H^{1}(\mathrm{~T}, \mathbb{Z})^{\Pi}$ is the subgroup of $H^{1}(\mathrm{~T}, \mathbb{Z})$ of elements fixed under the induced action of $\Pi$. By the Universal Coefficients 
Theorem, $H^{1}(\Pi, \mathbb{Z}) \cong \operatorname{Hom}\left(H_{1}(\Pi), \mathbb{Z}\right)=0$ and $\beta_{1}=\operatorname{rank}\left(H^{1}(\Gamma, \mathbb{Z})\right)$. The group $H^{2}(\Pi, \mathbb{Z})$ is finite by Proposition 5.3 in Chapter IV of Mac Lane [12]. Hence $\beta_{1}=$ $\operatorname{rank}\left(H^{1}(\mathrm{~T}, \mathbb{Z})^{\Pi}\right)$. By the Universal Coefficients Theorem,

$$
H^{1}(\mathrm{~T}, \mathbb{Z})^{\Pi} \cong \operatorname{Hom}\left(H_{1}(\mathrm{~T}), \mathbb{Z}\right)^{\Pi} \cong \mathrm{T}^{\Pi}=Z(\Gamma) .
$$

Thus $\operatorname{rank}(Z(\Gamma))=\beta_{1}$.

Let $V=\operatorname{Span}(Z(\Gamma))$. If $a+I \in Z(\Gamma)$ and $b+B \in \Gamma$, then

$$
B a+I=(b+B)(a+I)(b+B)^{-1}=a+I .
$$

Hence $V \subseteq \operatorname{Fix}(B)$. Therefore $\overline{Z(\Gamma)}=\{a+I \in \Gamma: a \in V\}=Z(\Gamma)$.

Let $Z(\Gamma)$ be the center of an $n$-dimensional space group $\Gamma$. Then every element of $Z(\Gamma)$ is a translation, the rank of $Z(\Gamma)$ is the first Betti number $\beta_{1}$ of $\Gamma$, and $Z(\Gamma)$ is a complete normal subgroup of $\Gamma$ by Theorem 6 . Let $V=\operatorname{Span}(Z(\Gamma))$. By Theorem 4, the flat orbifold $E^{n} / \Gamma$ geometrically fibers over the flat orbifold $\left(E^{n} / V\right) /(\Gamma / Z(\Gamma))$ with generic fiber the flat $\beta_{1}$-torus $V / Z(\Gamma)$.

Suppose $x \in V^{\perp}$ and $b+B \in \Gamma_{V+x}$. Write $b=c+d$ with $c \in V$ and $d \in V^{\perp}$. Then $(b+B)(V+x)=V+d+B x$, and so $d+B x=x$. If $v \in V$, then

$$
(b+B)(v+x)=b+v+B x=c+v+x .
$$

Thus $\Gamma_{V+x}$ acts as a discrete group of translations on $V+x$. As $\Gamma_{V+x}$ contains $Z(\Gamma)$, we have that $(V+x) / \Gamma_{V+x}$ is a $\beta_{1}$-torus. Thus all the fibers of the fibration projection $\eta: E^{n} / \Gamma \rightarrow\left(E^{n} / V\right) /(\Gamma / Z(\Gamma))$ are $\beta_{1}$-tori,

The $\beta_{1}$-torus $V / Z(\Gamma)$, as an additive group, acts on $E^{n} / \Gamma$ by

$$
(Z(\Gamma) v)(\Gamma x)=\Gamma(v+x) .
$$

The projection from $(V+x) / Z(\Gamma)$ to $(V+x) / \Gamma_{V+x}$ is a covering projection for each $x \in V^{\perp}$. Therefore the action of $V / Z(\Gamma)$ on $E^{n} / \Gamma$ is an injective toral action in the sense of Conner and Raymond [5] when $\Gamma$ is torsion-free.

\section{Equivalence of geometric fibrations}

Let $M$ be a flat $n$-orbifold. Suppose $M$ geometrically fibers over a flat $m$-orbifold $B_{i}$ with generic fiber a flat $(n-m)$-orbifold $F_{i}$ and fibration projection $\eta_{i}: M \rightarrow B_{i}$ for $i=1,2$. Then the fibration projections $\eta_{1}$ and $\eta_{2}$ are said to be geometrically equivalent if there is an isometry $\beta: B_{1} \rightarrow B_{2}$ such that $\beta \eta_{1}=\eta_{2}$. 
Theorem 7 Let $\Gamma$ be an $n$-dimensional space group. Suppose that the flat orbifold $E^{n} / \Gamma$ geometrically fibers over a flat $m$-orbifold $B$ with generic fiber a connected flat $(n-m)$-orbifold $F$ and fibration projection $\eta: E^{n} / \Gamma \rightarrow B$. Then $\Gamma$ has a complete normal subgroup $\mathrm{N}$ such that $\eta$ is geometrically equivalent to the fibration projection determined by $\mathrm{N}$.

Proof The fibration projection $\eta$ is locally isometrically equivalent to a natural projection $(F \times D) / G \rightarrow D / G$, where $G$ is a finite group acting diagonally on $F \times D$, isometrically on $F$ and effectively and orthogonally on an open $m$-disk $D$. The fibers of the projection $(F \times D) / G \rightarrow D / G$ are connected, totally geodesic, and parallel, and so the fibers of $\eta$ are connected, totally geodesic, and parallel. Hence there is a vector subspace $V$ of $E^{n}$ such that each coset of $V$ in $E^{n}$ projects onto a fiber of $\eta$ and $\Gamma$ maps each coset of $V$ to a coset of $V$. Let $b+B \in \Gamma$. Then $(b+B) V=b+B V$, and so $B V=V$.

Let $V+x$ project to a generic fiber $F$ of $\eta$, that is, to a fiber of $\eta$ with $G=1$. By conjugating $\Gamma$ by $-x+I$, we may assume that $x=0$. Now $F=\Gamma V / \Gamma$ which is isomorphic to $V / \Gamma_{V}$ where $\Gamma_{V}=\{\gamma \in \Gamma: \gamma(V)=V\}$. As $\eta$ is isometrically equivalent to the projection $F \times D \rightarrow D$ in a tubular neighborhood of the fiber $F$, we deduce that

$$
\Gamma_{V}=\left\{a+A \in \Gamma: a \in V \text { and } V^{\perp} \subseteq \operatorname{Fix}(A)\right\} .
$$

We claim that $\Gamma_{V}$ is a normal subgroup of $\Gamma$. Let $b+B \in \Gamma$ and $a+A \in \Gamma_{V}$. Then we have

$$
(b+B)(a+A)(b+B)^{-1}=b+B a-B A B^{-1} b+B A B^{-1} .
$$

If $x \in V^{\perp}$, then $B^{-1} x \in V^{\perp}$, and so $B A B^{-1} x=x$. Therefore $V^{\perp} \subseteq \operatorname{Fix}\left(B A B^{-1}\right)$. Write $b=c+d$ with $c \in V$ and $d \in V^{\perp}$. Then we have

$$
\begin{aligned}
b+B a-B A B^{-1} b & =b+B a-B A B^{-1} c-B A B^{-1} d \\
& =b+B a-B A B^{-1} c-d \\
& =c+B a-B A B^{-1} c
\end{aligned}
$$

which is an element of $V$. Therefore $(b+B)(a+A)(b+B)^{-1} \in \Gamma_{V}$. Thus $\Gamma_{V}$ is a normal subgroup of $\Gamma$.

Now $F=V / \Gamma_{V}$ is compact, and so $\Gamma_{V}$ acts as a space group of isometries of $V$. Therefore $V=\operatorname{Span}\left(\Gamma_{V}\right)$ by Part (1) of Theorem 1. Hence $\bar{\Gamma}_{V}=\Gamma_{V}$.

The fibration projection $\eta$ and the fibration projection $\eta_{V}$ determined by $\Gamma_{V}$ have the same fibers. Hence there is a homeomorphism $\beta: B \rightarrow\left(E^{n} / V\right) /\left(\Gamma / \Gamma_{V}\right)$ such that 
$\beta \eta=\eta_{V}$. The map $\phi$ is an isometry, since the metrics on $B$ and $\left(E^{n} / V\right) /\left(\Gamma / \Gamma_{V}\right)$ are determined by the distance between fibers in $E^{n} / \Gamma$. Therefore $\eta$ is geometrically equivalent to $\eta_{V}$.

Let $M_{i}$ be a connected, complete, flat $n$-orbifold for $i=1,2$. Suppose $M_{i}$ geometrically fibers over a flat $m$-orbifold $B_{i}$ with generic fiber a flat $(n-m)$-orbifold $F_{i}$ and fibration projection $\eta_{i}: M_{i} \rightarrow B_{i}$ for $i=1,2$. The fibration projections $\eta_{1}$ and $\eta_{2}$ are said to be isometrically equivalent if there are isometries $\alpha: M_{1} \rightarrow M_{2}$ and $\beta: B_{1} \rightarrow B_{2}$ such that $\beta \eta_{1}=\eta_{2} \alpha$.

Theorem 8 Let $M$ be a compact, connected, flat $n$-orbifold. If $M$ geometrically fibers over a flat $m$-orbifold $B$ with generic fiber a flat $(n-m)$-orbifold $F$ and fibration projection $\eta: M \rightarrow B$, then there exists an $n$-dimensional space group $\Gamma$ and a complete normal subgroup $\mathrm{N}$ of $\Gamma$ such that $\eta$ is isometrically equivalent to the fibration projection $\eta_{V}: E^{n} / \Gamma \rightarrow\left(E^{n} / V\right) /(\Gamma / \mathrm{N})$ determined by $\mathrm{N}$.

Proof There exists an $n$-dimensional space group $\Gamma$ and an isometry $\alpha: M \rightarrow E^{n} / \Gamma$ by Theorem 13.3.10 of Ratcliffe[14]. The map $\eta \alpha^{-1}: E^{n} / \Gamma \rightarrow B$ is a geometric fibration projection. There exists a complete normal subgroup $\mathrm{N}$ of $\Gamma$ and an isometry $\beta: B \rightarrow\left(E^{n} / V\right) /(\Gamma / \mathrm{N})$ such that $\beta\left(\eta \alpha^{-1}\right)=\eta_{V}$ by Theorem 7. Hence $\beta \eta=\eta_{V} \alpha$, and so $\eta$ is isometrically equivalent to $\eta_{V}$.

Theorem 9 Let $\mathrm{N}_{i}$ be a complete normal subgroup of an $n$-dimensional crystallographic group $\Gamma_{i}$ for $i=1,2$, and let $\eta_{i}: E^{n} / \Gamma_{i} \rightarrow\left(E^{n} / V_{i}\right) /\left(\Gamma_{i} / \mathrm{N}_{i}\right)$ be the fibration projections determined by $\mathrm{N}_{i}$ for $i=1,2$. Then $\eta_{1}$ and $\eta_{2}$ are isometrically equivalent if and only if there is an isometry $\xi$ of $E^{n}$ such that $\xi \Gamma_{1} \xi^{-1}=\Gamma_{2}$ and $\xi \mathrm{N}_{1} \xi^{-1}=\mathrm{N}_{2}$.

Proof Suppose $\eta_{1}$ and $\eta_{2}$ are isometrically equivalent. Then there exists isometries $\alpha: E^{n} / \Gamma_{1} \rightarrow E^{n} / \Gamma_{2}$ and $\beta:\left(E^{n} / V_{1}\right) /\left(\Gamma / \mathrm{N}_{1}\right) \rightarrow\left(E^{n} / V_{2}\right) /\left(\Gamma / \mathrm{N}_{2}\right)$ such that $\beta \eta_{1}=$ $\eta_{2} \alpha$. The isometry $\alpha$ lifts to an isometry $\xi$ of $E^{n}$ such that $\alpha\left(\Gamma_{1} x\right)=\Gamma_{2} \xi(x)$ for each $x \in E^{n}$ by Theorem 13.2.6 of Ratcliffe [14]. Therefore $\xi \Gamma_{1} \xi^{-1}=\Gamma_{2}$. As $\beta \eta_{1}=\eta_{2} \alpha$, the isometry $\alpha$ maps a generic fiber of $\eta_{1}$ onto a generic fiber of $\eta_{2}$. Let $V_{1}+x$ be a coset of $V_{1}$ that projects to a generic fiber of $\eta_{1}$. Then $\xi$ maps $V_{1}+x$ onto $V_{2}+\xi(x)$ and $V_{2}+\xi(x)$ projects to a generic fiber of $\eta_{2}$. Hence $\xi$ conjugates the stabilizer of $V_{1}+x$ in $\Gamma_{1}$ to the stabilizer of $V_{2}+\xi(x)$ in $\Gamma_{2}$. Therefore $\xi \mathrm{N}_{1} \xi^{-1}=\mathrm{N}_{2}$.

Conversely, suppose $\xi$ is an isometry of $E^{n}$ such that $\xi \Gamma_{1} \xi^{-1}=\Gamma_{2}$ and $\xi \mathrm{N}_{1} \xi^{-1}=\mathrm{N}_{2}$. Then $\xi$ induces an isometry $\alpha: E^{n} / \Gamma_{1} \rightarrow E^{n} / \Gamma_{2}$ defined by $\alpha\left(\Gamma_{1} x\right)=\Gamma_{2} \xi(x)$ for each $x \in E^{n}$. Let $\mathrm{T}_{i}$ be the group of translations of $\Gamma_{i}$ for $i=1,2$. Then $\xi \mathrm{T}_{1} \xi^{-1}=\mathrm{T}_{2}$, 
since $\mathrm{T}_{i}$ is the unique maximal abelian normal subgroup of $\Gamma_{i}$ for $i=1,2$. Hence $\xi\left(\mathrm{N}_{1} \cap \mathrm{T}_{1}\right) \xi^{-1}=\mathrm{N}_{2} \cap \mathrm{T}_{2}$, and so if $\xi=b+B$, then $B V_{1}=V_{2}$. Therefore $\xi$ maps each coset of $V_{1}$ in $E^{n}$ onto a coset of $V_{2}$ in $E^{n}$. Hence $\xi$ induces an isometry $\bar{\xi}: E^{n} / V_{1} \rightarrow E^{n} / V_{2}$ defined $\bar{\xi}\left(V_{1}+x\right)=V_{2}+\xi(x)$. If $\gamma \in \Gamma_{1}$ and $x \in E^{n}$, then we have

$$
\begin{aligned}
\bar{\xi}\left(\mathrm{N}_{1} \gamma\left(V_{1}+x\right)\right) & =\bar{\xi}\left(V_{1}+\gamma(x)\right) \\
& =V_{2}+\xi \gamma(x) \\
& =V_{2}+\xi \gamma \xi^{-1} \xi(x)=\left(\mathrm{N}_{2} \xi \gamma \xi^{-1}\right) \bar{\xi}\left(V_{1}+x\right)
\end{aligned}
$$

Hence $\bar{\xi}\left(\mathrm{N}_{1} \gamma\right)=\left(\mathrm{N}_{2} \xi \gamma \xi^{-1}\right) \bar{\xi}$, and so $\bar{\xi}\left(\mathrm{N}_{1} \gamma\right) \bar{\xi}^{-1}=\mathrm{N}_{2} \xi \gamma \xi^{-1}$. Therefore we have $\bar{\xi}\left(\Gamma_{1} / \mathrm{N}_{1}\right) \bar{\xi}^{-1}=\Gamma_{2} / \mathrm{N}_{2}$. Hence $\bar{\xi}$ induces an isometry

$$
\beta:\left(E^{n} / V_{1}\right) /\left(\Gamma_{1} / \mathrm{N}_{1}\right) \rightarrow\left(E^{n} / V_{2}\right) /\left(\Gamma_{2} / \mathrm{N}_{2}\right)
$$

defined by

$$
\beta\left(\left(\Gamma_{1} / \mathrm{N}_{1}\right)\left(V_{1}+x\right)\right)=\left(\Gamma_{2} / \mathrm{N}_{2}\right) \bar{\xi}\left(V_{1}+x\right)=\left(\Gamma_{2} / \mathrm{N}_{2}\right)\left(V_{2}+\xi(x)\right) .
$$

If $x \in E^{n}$, we have

$$
\begin{aligned}
\beta \eta_{1}\left(\Gamma_{1} x\right) & =\beta\left(\left(\Gamma_{1} / \mathrm{N}_{1}\right)\left(V_{1}+x\right)\right) \\
& =\left(\Gamma_{2} / \mathrm{N}_{2}\right)\left(V_{2}+\xi(x)\right) \\
& =\eta_{2}\left(\Gamma_{2} \xi(x)\right)=\eta_{2} \alpha\left(\Gamma_{1} x\right) .
\end{aligned}
$$

Therefore $\beta \eta_{1}=\eta_{2} \alpha$. Thus $\eta_{1}$ and $\eta_{2}$ are isometrically equivalent.

Let $M$ be a connected, complete flat $n$-orbifold. A universal orbifold covering projection is a geometric fibration projection $\pi: \widetilde{M} \rightarrow M$ that is isometrically equivalent to the natural projection $\pi_{\Gamma}: E^{n} \rightarrow E^{n} / \Gamma$ for some discrete group $\Gamma$ of isometries of $E^{n}$. There exists a universal orbifold covering projection $\pi: \widetilde{M} \rightarrow M$ by [14, Theorem 13.3.10], and any two universal orbifold covering projections $\pi_{i}: \widetilde{M}_{i} \rightarrow M$, $i=1,2$, are isometrically equivalent by [14, Theorem 13.2.6].

Let $\pi_{i}: \widetilde{M}_{i} \rightarrow M_{i}$ be universal orbifold covering projections for $i=1,2$. A homeomorphism $\alpha: M_{1} \rightarrow M_{2}$ is said to be affine if there is an affine homeomorphism $\tilde{\alpha}: \widetilde{M}_{1} \rightarrow \widetilde{M}_{2}$ such that $\alpha \pi_{1}=\pi_{2} \widetilde{\alpha}$. Note that $\alpha: M_{1} \rightarrow M_{2}$ being affine does not depend on the choice of the universal orbifold covering projections $\pi_{i}: \widetilde{M}_{i} \rightarrow M_{i}$.

The fibration projections $\eta_{1}$ and $\eta_{2}$ are said to be affinely equivalent if there are affine homeomorphisms $\alpha: M_{1} \rightarrow M_{2}$ and $\beta: B_{1} \rightarrow B_{2}$ such that $\beta \eta_{1}=\eta_{2} \alpha$. 
Theorem 10 Let $\mathrm{N}_{i}$ be a complete normal subgroup of an $n$-dimensional space group $\Gamma_{i}$ for $i=1,2$, and let $\eta_{i}: E^{n} / \Gamma_{i} \rightarrow\left(E^{n} / V_{i}\right) /\left(\Gamma_{i} / \mathrm{N}_{i}\right)$ be the fibration projections determined by $\mathrm{N}_{i}$ for $i=1,2$. Then the following are equivalent:

(1) The fibration projections $\eta_{1}$ and $\eta_{2}$ are affinely equivalent.

(2) There is an affine homeomorphism $\phi$ of $E^{n}$ such that $\phi \Gamma_{1} \phi^{-1}=\Gamma_{2}$ and $\phi \mathrm{N}_{1} \phi^{-1}=\mathrm{N}_{2}$.

(3) There is an isomorphism $\psi: \Gamma_{1} \rightarrow \Gamma_{2}$ such that $\psi\left(\mathrm{N}_{1}\right)=\mathrm{N}_{2}$.

Proof The proof of the equivalence of (1) and (2) is the same as the proof of Theorem 9. The equivalence of (2) and (3) follows from Part (2) of Theorem 1.

\section{Reducibility of crystallographic groups}

Let $\mathrm{N}$ be a normal subgroup of an $n$-dimensional space group $\Gamma$. The dimension of $\mathrm{N}$, denoted by $\operatorname{dim}(\mathrm{N})$, is defined to be the dimension of $V=\operatorname{Span}(\mathrm{N})$. Note that $\operatorname{dim}(\mathrm{N})$ is equal to (1) the rank of the translation subgroup of $N,(2)$ the Hirsch length of $\mathrm{N}$, and (3) the virtual cohomological dimension of $\mathrm{N}$ by Theorem 2 . The theory in this paper is nontrivial only if $0<\operatorname{dim}(\mathrm{N})<n$, and so we should discuss when such a normal subgroup $\mathrm{N}$ exists.

Let $\mathrm{T}$ be the group of translations of $\Gamma$, and let $\Pi$ be the point group of $\Gamma$. The group $\mathrm{T}$ is free abelian of rank $n$ and $\{b: b+I \in \mathrm{T}\}$ spans $E^{n}$ by Theorem 1 . Choose a set $\left\{b_{1}+I, \ldots, b_{n}+I\right\}$ of $n$ generators of T. Then $\left\{b_{1}, \ldots, b_{n}\right\}$ is a basis of $E^{n}$. Let $\gamma=b+B \in \Gamma$. Then $\gamma\left(b_{j}+I\right) \gamma^{-1}=B b_{j}+I \in \mathrm{T}$ for each $j=1, \ldots, n$. Hence there are integers $c_{i j}$ for $i, j=1, \ldots, n$ such that $B b_{j}=\sum_{i=1}^{n} c_{i j} b_{i}$ for each $j$. The representation $\rho: \Pi \rightarrow \operatorname{GL}(n, \mathbb{Z})$ defined by $\rho(B)=\left(c_{i j}\right)$ is faithful. The representation $\rho$ is said be reducible if there is an integer $k$, with $0<k<n$, such that every matrix in the image of $\rho$ is in the block form

$$
\left(\begin{array}{ll}
A & B \\
O & D
\end{array}\right)
$$

where $\mathrm{A}$ is a $k \times k$ block and $\mathrm{O}$ is a $(n-k) \times k$ block of zeros.

The group $\Gamma$ is said to be $\mathbb{Z}$-reducible, if there is a set of $n$ generators of $\mathrm{T}$ such that the corresponding representation $\rho: \Pi \rightarrow \operatorname{GL}(n, \mathbb{Z})$ is reducible. 
Theorem 11 Let $\Gamma$ be an $n$-dimensional space group with translation group $\mathrm{T}$ and point group $\Pi$. Then the following are equivalent:

(1) The group $\Gamma$ is $\mathbb{Z}$-reducible.

(2) There exists a $\Pi$-invariant vector subspace $V$ of $E^{n}$ with basis $\left\{b_{1}, \ldots, b_{k}\right\}$ such that $0<k<n$ and $b_{i}+I \in \mathrm{T}$ for each $i=1, \ldots, k$.

(3) The group $\Gamma$ has a normal subgroup $\mathrm{N}$ of dimension $k$ with $0<k<n$.

Proof Suppose $\Gamma$ is $\mathbb{Z}$-reducible. Then there is a set of generators $\left\{b_{1}+I, \ldots, b_{n}+I\right\}$ of $\mathrm{T}$ such that the corresponding representation $\rho: \Pi \rightarrow \operatorname{GL}(n, \mathbb{Z})$ is reducible. Let $k$ be the integer in the definition of reducibility, and let $V=\operatorname{Span}\left\{b_{1}, \ldots, b_{k}\right\}$. Then $V$ is a $\Pi$-invariant vector subspace of $E^{n}$ with basis $\left\{b_{1}, \ldots, b_{k}\right\}$ such that $0<k<n$ and $b_{i}+I \in \mathrm{T}$ for each $i=1, \ldots, k$. Thus (1) implies (2).

Suppose there exists a $\Pi$-invariant vector subspace $V$ of $E^{n}$ with basis $\left\{b_{1}, \ldots, b_{k}\right\}$ such that $0<k<n$ and $b_{i}+I \in \mathrm{T}$ for each $i=1, \ldots, k$. Define

$$
\mathrm{N}=\{a+I \in \mathrm{T}: a \in V\} .
$$

As $V$ is $\Pi$-invariant, $\mathrm{N}$ is a normal subgroup of $\Gamma$. As $V=\operatorname{Span}(\mathrm{N})$, we have that $\operatorname{dim}(\mathrm{N})=k$. Thus (2) implies (3).

Suppose $\Gamma$ has a normal subgroup $\mathrm{N}$ of dimension $k$ with $0<k<n$. Then $\mathrm{T}$ has a set of generators $\left\{b_{1}+I, \ldots, b_{n}+I\right\}$ such that $b_{1}+I, \ldots, b_{k}+I$ generate $\overline{\mathrm{N}} \cap \mathrm{T}$ by the proof of Lemma 1 . As $\overline{\mathrm{N}} \cap \mathrm{T}$ is a normal subgroup of $\Gamma$, the additive group generated by $b_{1}, \ldots, b_{k}$ is $\Pi$-invariant. Hence the representation $\rho: \Pi \rightarrow \operatorname{GL}(n, \mathbb{Z})$ determined by the set of generators $\left\{b_{1}+I, \ldots, b_{n}+I\right\}$ of $\mathrm{T}$ is reducible. Thus (3) implies (1).

Theorem 12 Let $\Gamma$ be an $n$-dimensional space group. If $\Gamma$ has a normal subgroup $\mathrm{N}$ of dimension $k$, then $\Gamma$ has a normal subgroup $\mathrm{N}^{\prime}$ of dimension $n-k$.

Proof This follows from Theorem 11 and Proposition 2.1.1 of Brown, Neubüser and Zassenhaus [4].

\section{Geometric fiber bundles}

Let $\Gamma$ be an $n$-dimensional space group. If $E^{n} / \Gamma$ geometrically fibers over a flat manifold $B$ with generic fiber a flat orbifold $F$, then $E^{n} / \Gamma$ is a fiber bundle over $B$ with totally geodesic fibers isometric to $F$. 
Definition A flat $n$-orbifold $M$ is a geometric fiber bundle over a flat $m$-manifold $B$ with fiber a flat $(n-m)$-orbifold $F$ if there is a surjective map $\eta: M \rightarrow B$, called the fibration projection, such that for each point $y$ in $B$, there is an open metric ball $B(y, r)$ of radius $r>0$ centered at $y$ in $B$ such that $\eta$ is isometrically equivalent on $\eta^{-1}(B(y, r))$ to the natural projection $F \times B_{y} \rightarrow B_{y}$ onto an open metric ball $B_{y}$ in $E^{m}$ of radius $r$.

Lemma 2 Let $\mathrm{N}$ be a complete normal subgroup of an $n$-dimensional space group $\Gamma$, and let $V=\operatorname{Span}(\mathrm{N})$. Then $\left(E^{n} / V\right) /(\Gamma / \mathrm{N})$ is a flat manifold if and only if $\Gamma / \mathrm{N}$ is torsion-free.

Proof Suppose $\left(E^{n} / V\right) /(\Gamma / \mathrm{N})$ is a flat manifold. Then the quotient map from $E^{n} / V$ to $\left(E^{n} / V\right) /(\Gamma / \mathrm{N})$ is a universal covering projection with $\Gamma / \mathrm{N}$ its group of covering transformations. Therefore $\left(E^{n} / V\right) /(\Gamma / \mathrm{N})$ is an aspherical manifold, and so its fundamental group is torsion-free. Therefore $\Gamma / \mathrm{N}$ is torsion-free.

Conversely if $\Gamma / \mathrm{N}$ is torsion-free, then $\left(E^{n} / V\right) /(\Gamma / \mathrm{N})$ is a flat manifold, since the finite group $G_{x}=\Gamma_{V+x} / \mathrm{N}$ is trivial for each $x \in V^{\perp}$.

Theorem 13 Let $\mathrm{N}$ be a normal subgroup of an $n$-dimensional space group $\Gamma$ such that $\Gamma / \mathrm{N}$ is torsion-free, and let $V=\operatorname{Span}(\mathrm{N})$. Then $\mathrm{N}$ is complete, and the flat orbifold $E^{n} / \Gamma$ is a geometric fiber bundle over the flat manifold $\left(E^{n} / V\right) /(\Gamma / \mathrm{N})$ with fiber the flat orbifold $V / \mathrm{N}$.

Proof We have that $\mathrm{N}$ is complete by Theorem 5. The rest of the theorem follows from Lemma 2 and Theorem 4.

Theorem 14 Let $\Gamma$ be an $n$-dimensional space group with first Betti number $\beta_{1}$. Then $\Gamma$ has a unique normal subgroup $\mathrm{N}$ such that $\Gamma / \mathrm{N}$ is a free abelian group of rank $\beta_{1}$, and the flat orbifold $E^{n} / \Gamma$ uniquely fibers as a geometric fiber bundle over a flat $\beta_{1}$-torus.

Proof We have $\Gamma /[\Gamma, \Gamma] \cong G \oplus \mathbb{Z}^{\beta_{1}}$ with $G$ a finite abelian group. Hence the subgroup of $\Gamma$ containing $[\Gamma, \Gamma]$ that corresponds to $G$ is the unique normal subgroup $\mathrm{N}$ of $\Gamma$ such that $\Gamma / \mathrm{N}$ is a free abelian group of rank $\beta_{1}$. By Theorem 13, we have that $\mathrm{N}$ is complete. Let $V=\operatorname{Span}(\mathrm{N})$. Then $\left(E^{n} / V\right) /(\Gamma / \mathrm{N})$ is a flat $\beta_{1}$-torus, since $\Gamma / \mathrm{N}$ is a free abelian group of rank $\beta_{1}$. Therefore $E^{n} / \Gamma$ fibers as a geometric fiber bundle over a flat $\beta_{1}$-torus by Theorem 4 . The flat orbifold $E^{n} / \Gamma$ uniquely fibers as a geometric fiber bundle over a flat $\beta_{1}$-torus by Theorem 7 , since $\mathrm{N}$ is the unique normal subgroup of $\Gamma$ such that $\Gamma / \mathrm{N}$ is free abelian of rank $\beta_{1}$. 
Lemma 3 If $\Gamma$ is an $n$-dimensional space group with translation group $\mathrm{T}$ and point group $\Pi$, then the transfer homomorphism $\operatorname{tr}: \Gamma \rightarrow \mathrm{T}$ is given by the formula

$$
\operatorname{tr}(b+B)=\left(\sum\{A: A \in \Pi\}\right) b+I \text { for each } b+B \in \Gamma .
$$

Proof For each $A \in \Pi$, choose a coset representative $a_{A}+A$ of $\mathrm{T}$ in $\Gamma$ corresponding to $A$. Given an element $b+B \in \Gamma$ and a coset representative $a_{A}+A$, then there exists a unique coset representative $a_{A^{\prime}}+A^{\prime}$ such that

$$
\left(a_{A}+A\right)(b+B)\left(a_{A^{\prime}}+A^{\prime}\right)^{-1} \in \mathrm{T} .
$$

The transfer homomorphism tr: $\Gamma \rightarrow \mathrm{T}$ is defined by the formula

$$
\operatorname{tr}(b+B)=\prod\left\{\left(a_{A}+A\right)(b+B)\left(a_{A^{\prime}}+A^{\prime}\right)^{-1}: A \in \Pi\right\} .
$$

We have that

$$
\left(a_{A}+A\right)(b+B)\left(a_{A^{\prime}}+A^{\prime}\right)^{-1}=a_{A}+A b-A B\left(A^{\prime}\right)^{-1} a_{A^{\prime}}+A B\left(A^{\prime}\right)^{-1} .
$$

Therefore $A B\left(A^{\prime}\right)^{-1}=I$, and so $A^{\prime}=A B$. Hence we have that

$$
\begin{aligned}
\operatorname{tr}(b+B) & =\prod\left\{a_{A}+A b-a_{A B}+I: A \in \Pi\right\} \\
& =\left(\sum\left\{a_{A}: A \in \Pi\right\}-\sum\left\{a_{A B}: A \in \Pi\right\}+\sum\{A b: A \in \Pi\}\right)+I \\
& =\left(\sum\{A: A \in \Pi\}\right) b+I .
\end{aligned}
$$

Lemma 4 If $\Pi$ is a finite group of orthogonal transformations of $E^{n}$, then

(1) $\operatorname{Im}\left(\sum\{A \in \Pi\}\right)=\operatorname{Fix}(\Pi)$,

(2) $\operatorname{Ker}\left(\sum\{A \in \Pi\}\right)=\operatorname{Fix}(\Pi)^{\perp}$.

Proof Let $B \in \Pi$. Observe that

$$
(B-I)\left(\sum\{A \in \Pi\}\right)=O .
$$

Now $\operatorname{Ker}(B-I)=\operatorname{Fix}(B)$. Hence we have

$$
\operatorname{Im}\left(\sum\{A \in \Pi\}\right) \subseteq \operatorname{Fix}(\Pi) .
$$

Let $x \in \operatorname{Fix}(\Pi)$. Then we have

$$
\left(\sum\{A \in \Pi\}\right)(x)=|\Pi| x .
$$

Hence $x \in \operatorname{Im}\left(\sum\{A \in \Pi\}\right)$. This proves (1).

Let $x \in \operatorname{Ker}\left(\sum\{A \in \Pi\}\right)$. Write $x=u+v$ with $u \in \operatorname{Fix}(\Pi)$ and $v \in \operatorname{Fix}(\Pi)^{\perp}$. Then we have that

$$
0=\left(\sum\{A \in \Pi\}\right)(x)=|\Pi| u+\left(\sum\{A \in \Pi\}\right)(v) .
$$


Now $|\Pi| u \in \operatorname{Fix}(\Pi)$ and $\left(\sum\{A \in \Pi\}\right)(v) \in \operatorname{Fix}(\Pi)^{\perp}$, since $\operatorname{Fix}(\Pi)^{\perp}$ is a $\Pi$-invariant subspace of $E^{n}$. Therefore $|\Pi| u=0$, and so $u=0$. Hence $x \in \operatorname{Fix}(\Pi)^{\perp}$.

Conversely, suppose $x \in \operatorname{Fix}(\Pi)^{\perp}$. By (1), we have

$$
\left(\sum\{A \in \Pi\}\right)(x) \in \operatorname{Fix}(\Pi) \cap \operatorname{Fix}(\Pi)^{\perp}=\{0\} .
$$

Therefore $x \in \operatorname{Ker}\left(\sum\{A \in \Pi\}\right)$. This proves (2).

Let $\mathrm{N}$ and $\mathrm{N}^{\prime}$ be a normal subgroups of a space group $\Gamma$. We say that $\mathrm{N}$ and $\mathrm{N}^{\prime}$ are orthogonal if $\operatorname{Span}\left(\mathrm{N}^{\prime}\right)=(\operatorname{Span}(\mathrm{N}))^{\perp}$. If $\mathrm{N}$ and $\mathrm{N}^{\prime}$ are orthogonal, complete, normal subgroups of $\Gamma$, we define $\mathrm{N}^{\perp}=\mathrm{N}^{\prime}$.

Theorem 15 Let $\Gamma$ be an $n$-dimensional space group with first Betti number $\beta_{1}$. Then the kernel of the transfer homomorphism $\mathrm{tr}: \Gamma \rightarrow \mathrm{T}$ is the unique normal subgroup $\mathrm{N}$ of $\Gamma$ such that $\Gamma / \mathrm{N}$ is a free abelian group of rank $\beta_{1}$, Moreover $\mathrm{N}$ and $Z(\Gamma)$ are orthogonal, complete, normal subgroups of $\Gamma$.

Proof Let $\Pi$ be the point group of $\Gamma$. By Lemmas 3 and 4, we have that

$$
\operatorname{tr}(Z(\Gamma))=\{|\Pi| b+I: b+I \in Z(\Gamma)\} \subseteq \operatorname{Im}(\operatorname{tr}) \subseteq Z(\Gamma) .
$$

Hence $\operatorname{Im}(\mathrm{tr})$ is a free abelian group of $\operatorname{rank} \beta_{1}$ by Theorem 6 . Therefore $\operatorname{Ker}(\operatorname{tr})$ is the unique normal subgroup $\mathrm{N}$ of $\Gamma$ such that $\Gamma / \mathrm{N}$ is a free abelian group of rank $\beta_{1}$.

By Lemmas 3 and 4, we have that

$$
\mathrm{N}=\operatorname{Ker}(\operatorname{tr})=\left\{b+B \in \Gamma: b \in \operatorname{Fix}(\Pi)^{\perp}\right\} .
$$

Hence $\operatorname{Span}(\mathrm{N}) \subseteq \operatorname{Fix}(\Pi)^{\perp}$. By Theorems Theorem 6 and Theorem 14, we have that

$$
\operatorname{dim}(\operatorname{Span}(\mathrm{N}))=n-\beta_{1}=\operatorname{dim}\left(\operatorname{Fix}(\Pi)^{\perp}\right) .
$$

Therefore $\operatorname{Span}(\mathrm{N})=\operatorname{Fix}(\Pi)^{\perp}$. Now as $\operatorname{Span}(Z(\Gamma))=\operatorname{Fix}(\Pi)$, we conclude that $\mathrm{N}^{\perp}=Z(\Gamma)$.

\section{Crystallographic group extensions}

Let $\mathrm{N}$ be a complete normal subgroup of an $n$-dimensional space group $\Gamma$, let $V=$ $\operatorname{Span}(\mathrm{N})$, and let $m=\operatorname{dim}\left(E^{n} / V\right)$. Then $\mathrm{N}$ is an $(n-m)$-dimensional space group by Theorem 2 , and $\Gamma / \mathrm{N}$ is an $m$-dimensional space group by Theorem 4 . We call the exact sequence of natural group homomorphisms,

$$
1 \rightarrow \mathrm{N} \stackrel{i}{\longrightarrow} \Gamma \stackrel{p}{\longrightarrow} \Gamma / \mathrm{N} \rightarrow 1
$$


a space group extension. In this section, we study the relationship between the point groups of $\mathrm{N}, \Gamma$ and $\Gamma / \mathrm{N}$.

Lemma 5 Let $\Gamma$ be a space group with group of translations $\mathrm{T}$ and point group $\Pi$. Let $\mathrm{N}$ be a normal subgroup of $\Gamma$, and let $\Phi=\{A \in \Pi: a+A \in \mathrm{N}$ for some $a\}$. Then

(1) the group of translations of $\mathrm{N}$ is $\mathrm{N} \cap \mathrm{T}$,

(2) the point group of $\mathrm{N}$ is isomorphic to $\Phi$,

(3) the group $\Phi$ is a normal subgroup of $\Pi$.

Proof Let $V=\operatorname{Span}(\mathrm{N})$, and let $a+A \in \mathrm{N}$. By Theorem 2, we have that $a \in V$ and $V^{\perp} \subseteq \operatorname{Fix}(A)$. Hence $a+A \in \mathrm{N}$ acts as a translation on $V$ if and only if $A=I$. Thus (1) holds. Let $\eta: \Gamma \rightarrow \Pi$ be defined by $\eta(b+B)=B$. Then (2) follows from (1), since the $\operatorname{Ker}\left(\left.\eta\right|_{\mathrm{N}}\right)=\mathrm{N} \cap \mathrm{T}$, and (3) follows from (1), since $\mathrm{N} /(\mathrm{N} \cap \mathrm{T}) \cong \mathrm{NT} / \mathrm{T}$, and so $\Phi$ corresponds to the normal subgroup NT/T of $\Gamma / T$.

Theorem 16 Let $\Gamma$ be a space group with point group $\Pi$. Let $\mathrm{N}$ be a complete normal subgroup of $\Gamma$, let $V=\operatorname{Span}(\mathrm{N})$, let $\Psi=\left\{B \in \Pi: V^{\perp} \subseteq \operatorname{Fix}(B)\right\}$, let $\mathbf{M}=\{b+B \in \Gamma: B \in \Psi\}$, and let $\Phi$ be as in Lemma 5. Then

(1) the group of translations of $\Gamma / \mathrm{N}$ is $\mathrm{M} / \mathrm{N}$,

(2) the group $\Phi$ is a normal subgroup of $\Psi$,

(3) the group $\Psi$ is a normal subgroup of $\Pi$,

(4) the point group of $\Gamma / \mathrm{N}$ is isomorphic to $\Pi / \Psi$.

Proof Let $b+B \in \Gamma$. Suppose $b+B$ acts as a translation on $E^{n} / V$. Then $(b+B)(V+x)=V+c+x$ for some $c \in E^{n}$ for all $x \in V^{\perp}$. Now $(b+B)(V+x)=$ $V+b+B x$. Taking $x=0$, we see that $b-c \in V$, and so $V+b+B x=V+b+x$. Hence $B x-x \in V \cap V^{\perp}=\{0\}$. Therefore $x \in \operatorname{Ker}(B-I)=\operatorname{Fix}(B)$. Hence $V^{\perp} \subseteq \operatorname{Fix}(B)$, and so $b+B \in M$. Conversely if $b+B \in M$, then $b+B$ obviously acts as a translation on $E^{n} / V$. Thus (1) holds. Let $A \in \Phi$. By Theorem 2, we have that $A \in \Psi$. Thus (2) holds by Lemma 5 .

Let $B \in \Psi$, and let $C \in \Pi$. By Theorem 2, we have that $C$ leaves $V$ invariant. Hence $C$ leaves $V^{\perp}$ invariant. Therefore $C B C^{-1} \in \Psi$. Thus (3) holds.

Let $\mathrm{T}$ be the translation group of $\Gamma$. Then (4) follows from (1), since

$$
(\Gamma / \mathrm{N}) /(\mathrm{M} / \mathrm{N}) \cong \Gamma / \mathrm{M} \cong(\Gamma / \mathrm{T}) /(\mathrm{M} / \mathrm{T}) \cong \Pi / \Psi .
$$


Example Let $e_{1}=(1,0)$ and $e_{2}=(0,1)$, and let $t_{i}=e_{i}+I$ for $i=1,2$. Let $\beta=\frac{1}{2} e_{1}+\operatorname{diag}(1,-1)$, and let $\Gamma=\left\langle t_{1}, t_{2}, \beta\right\rangle$. Then $\Gamma$ is a 2 -dimensional space group, and $E^{2} / \Gamma$ is a flat Klein bottle. The group $\left\langle t_{2}\right\rangle$ is a complete normal subgroup of $\Gamma$ and $\operatorname{Span}\left\langle t_{2}\right\rangle=\operatorname{Span}\left\{e_{2}\right\}$. Hence $\Phi$ is trivial and $\Psi$ has order two.

Corollary 3 If $\Gamma$ is a space group with translation group $\mathrm{T}$, then the group of translations of $\Gamma / Z(\Gamma)$ is $\mathrm{T} / Z(\Gamma)$ and the point group of $\Gamma / Z(\Gamma)$ is isomorphic to the point group of $\Gamma$.

\section{Splitting crystallographic group extensions}

Let $\mathrm{N}$ be a complete normal subgroup of an $n$-dimensional space group $\Gamma$, let $V=$ $\operatorname{Span}(\mathrm{N})$, and consider the corresponding space group extension

$$
1 \rightarrow \mathrm{N} \stackrel{i}{\longrightarrow} \Gamma \stackrel{p}{\longrightarrow} \Gamma / \mathrm{N} \rightarrow 1 .
$$

In this section, we study the relationship between the above space group extension splitting ( $p$ having a right inverse) and the corresponding fibration projection $\eta: E^{n} / \Gamma \rightarrow\left(E^{n} / V\right) /(\Gamma / \mathrm{N})$ having an affine section. Here an affine section of $\eta$ is an affine map $\sigma:\left(E^{n} / V\right) /(\Gamma / \mathrm{N}) \rightarrow E^{n} / \Gamma$ such that $\eta \sigma$ is the identity map of $\left(E^{n} / V\right) /(\Gamma / \mathrm{N})$. A map $\sigma:\left(E^{n} / V\right) /(\Gamma / \mathrm{N}) \rightarrow E^{n} / \Gamma$ is affine if $\sigma$ lifts to an affine map $\tilde{\sigma}: E^{n} / V \rightarrow E^{n}$ with respect to the natural quotient maps.

Lemma 6 Let $\mathrm{N}$ be a complete normal subgroup of an $n$-dimensional space group $\Gamma$, and let $V=\operatorname{Span}(\mathrm{N})$. Let $\eta: E^{n} / \Gamma \rightarrow\left(E^{n} / V\right) /(\Gamma / \mathrm{N})$ be the fibration projection determined by $\mathrm{N}$. If the space group extension

$$
1 \rightarrow \mathrm{N} \stackrel{i}{\longrightarrow} \Gamma \stackrel{p}{\longrightarrow} \Gamma / \mathrm{N} \rightarrow 1
$$

splits, then $\eta$ has an affine section.

Proof Suppose that the space group extension splits. Then $\Gamma$ has a subgroup $\Sigma$ such that $p$ maps $\Sigma$ isometrically onto $\Gamma / \mathrm{N}$. By [14, Theorem 5.4.6], the group $\Sigma$ has a free abelian subgroup $\mathrm{H}$ of rank $m$ and finite index, there is an $m$-plane $Q$ of $E^{n}$ such that $\mathrm{H}$ acts effectively on $Q$ as a discrete group of translations, and the $m$-plane $Q$ is invariant under $\Sigma$. Hence

$$
m=\operatorname{dim}(\mathrm{H})=\operatorname{dim}(\Gamma / \mathrm{N})=\operatorname{dim}\left(E^{n} / V\right) .
$$

By conjugating $\Gamma$ by a translation, we may assume that $Q$ is a vector subspace of $E^{n}$. Let $a_{1}+A_{1}, \ldots, a_{m}+A_{m}$ be generators of $\mathrm{H}$. Then $a_{i} \in Q$ for each $i$ and $A_{i}$ 
fixes $Q$ pointwise for each $i$. Let $k$ be the order of the point group of $\Gamma$. Then $\left(a_{i}+A_{i}\right)^{k}=k a_{i}+I$ for each $i$. Hence, by replacing $\mathrm{H}$ by a subgroup of finite index, we may assume that $A_{i}=I$ for each $i$.

Now $p\left(a_{i}+I\right)$ acts on $E^{n} / V$ by $\mathrm{N}\left(a_{i}+I\right)(V+x)=V+x+a_{i}$, and so $p\left(a_{i}+I\right)$ acts as a translation on $E^{n} / V$ for each $i$. As $p(\mathrm{H})$ has finite index in $\Gamma / \mathrm{N}$, we have that $p(\mathrm{H})$ has finite index in the translation subgroup of $\Gamma / \mathrm{N}$. Therefore the vectors $V+a_{1}, \ldots, V+a_{m}$ span $E^{n} / V$. Hence the quotient map $\pi: E^{n} \rightarrow E^{n} / V$ maps $Q$ isomorphically onto $E^{n} / V$. Therefore $V \cap Q=\{0\}$.

If $x \in E^{n}$, then $x$ can be written uniquely as $x=x_{V}+x_{Q}$ with $x_{V} \in V$ and $x_{Q} \in Q$. Define $\phi: E^{n} / V \rightarrow Q$ by $\phi(V+x)=x_{Q}$. Then $\phi$ is a well-defined linear isomorphism.

Let $a+A \in \Sigma$. Then $a \in Q$ and $A$ leaves both $V$ and $Q$ invariant. Observe that

$$
\begin{aligned}
\phi(p(a+A)(V+x)) & =\phi(\mathrm{N}(a+A)(V+x)) \\
& =\phi(V+a+A x) \\
& =a+A x_{Q} \\
& =(a+A) x_{Q}=(a+A) \phi(V+x) .
\end{aligned}
$$

Hence $\phi$ induces an affine map $\bar{\phi}:\left(E^{n} / V\right) /(\Gamma / \mathrm{N}) \rightarrow E^{n} / \Gamma$ whose image is $\Gamma Q / \Gamma$. Observe that

$$
\begin{aligned}
\eta \bar{\phi}((\Gamma / \mathrm{N})(V+x)) & =\eta(\Gamma \phi(V+x)) \\
& =\eta\left(\Gamma x_{Q}\right) \\
& =(\Gamma / \mathrm{N})\left(V+x_{Q}\right)=(\Gamma / \mathrm{N})(V+x) .
\end{aligned}
$$

Therefore $\eta \bar{\phi}$ is the identity map. Thus $\bar{\phi}$ is an affine section of $\eta$.

Let $F$ be a connected flat $n$-orbifold. Then $F$ has a natural inner metric $d$. A point $x$ in $F$ is said to be an ordinary point of $F$ if there is an $r>0$ such that $B(x, r)=\{y \in F: d(x, y)<r\}$ is isometric to the open metric ball $B(0, r)$ in $E^{n}$.

Lemma 7 Let $\mathrm{N}$ be a complete normal subgroup of an $n$-dimensional space group $\Gamma$, and let $V=\operatorname{Span}(\mathrm{N})$. Let $\eta: E^{n} / \Gamma \rightarrow\left(E^{n} / V\right) /(\Gamma / \mathrm{N})$ be the fibration projection determined by $\mathrm{N}$. If $\eta$ has an affine section $\sigma:\left(E^{n} / V\right) /(\Gamma / \mathrm{N}) \rightarrow E^{n} / \Gamma$ such that $\operatorname{Im}(\sigma)$ intersects a fiber $F_{0}$ of $\eta$ at an ordinary point $x_{0}$ of $F_{0}$, then the space group extension $1 \rightarrow \mathrm{N} \rightarrow \Gamma \rightarrow \Gamma / \mathrm{N} \rightarrow 1$ splits. 
Proof By conjugating $\Gamma$ by a translation, we may assume that $x_{0}=\Gamma 0$. Then $\sigma$ lifts to an affine map $\tilde{\sigma}: E^{n} / V \rightarrow E^{n}$ such that $\operatorname{Im}(\widetilde{\sigma})$ is a vector subspace $Q$ of $E^{n}$ and the following diagram commutes

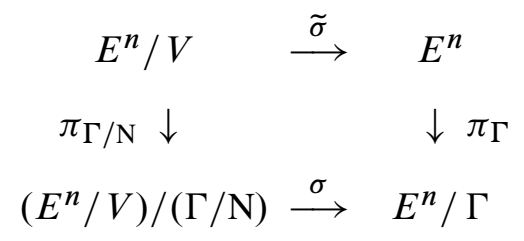

where the vertical maps are the quotient maps. Now we have

$$
\pi_{\Gamma}(Q)=\pi_{\Gamma}\left(\widetilde{\sigma}\left(E^{n} / V\right)\right)=\sigma\left(\pi_{\Gamma / \mathrm{N}}\left(E^{n} / V\right)\right)=\operatorname{Im}(\sigma) .
$$

Let $\tilde{\eta}: E^{n} \rightarrow E^{n} / V$ be the quotient map. Then $\pi_{\Gamma / \mathrm{N}} \tilde{\eta}=\eta \pi_{\Gamma}$, and so

$$
\pi_{\Gamma / \mathrm{N}} \tilde{\eta}(Q)=\eta \pi_{\Gamma}(Q)=\eta(\operatorname{Im}(\sigma))=\left(E^{n} / V\right) /(\Gamma / \mathrm{N}) .
$$

As $\tilde{\eta}(Q)$ is a vector subspace of $E^{n} / V$, we deduce that $\tilde{\eta}(Q)=E^{n} / V$. Therefore $\tilde{\eta} \widetilde{\sigma}: E^{n} / V \rightarrow E^{n} / V$ is an affine homeomorphism, and so $\tilde{\sigma}: E^{n} / V \rightarrow E^{n}$ is an affine embedding whose image is $Q$.

Let $\Sigma$ be the stabilizer of $Q$ in $\Gamma$, and let $a+A \in \mathrm{N} \cap \Sigma$. Then $a \in V \cap Q=\{0\}$. Hence $A=I$, since $\Gamma 0$ is an ordinary point of the fiber $F_{0}=\pi_{\Gamma}(V)$, which is isometric to $V / \Gamma_{V}$. Therefore $N \cap \Sigma=\{I\}$.

Suppose $a+A \in \Sigma$ and $a+A$ fixes $Q$ pointwise. Then $a=0$, since $0 \in Q$. Let $x \in Q$. Write $x=v+w$ with $v \in V$ and $w \in V^{\perp}$. Then $A x=A v+A w$, and so $x=A v+A w$. Now $A v \in V$ and $A w \in V^{\perp}$, and so $A v=v$ and $A w=w$. Hence $A$ fixes $V^{\perp}$ pointwise, since $\tilde{\eta}(Q)=E^{n} / V$. Therefore $A \in \mathrm{N}$. Hence $A=I$, since $\Gamma 0$ is an ordinary point of $F_{0}$. Thus $\Sigma$ acts effectively on $Q$.

Suppose $x \in Q$, and $\gamma \in \Gamma$, and $y=\gamma x \in Q$. Choose $r>0$ small enough so that $B(y, r) \cap B(\alpha y, r)=\varnothing$ unless $\alpha \in \Gamma_{y}$, the stabilizer of $y$ in $\Gamma$. Then we have

$$
\pi_{\Gamma}^{-1}\left(\pi_{\Gamma}(B(y, r) \cap Q)\right) \cap B(y, r)=\bigcup_{\alpha \in \Gamma_{y}} \alpha(B(y, r) \cap Q) .
$$

We have $\pi_{\Gamma}(B(y, r) \cap \gamma Q)=\pi_{\Gamma}(B(y, r) \cap Q)$. Therefore, we have

$$
B(y, r) \cap \gamma Q \subset \bigcup_{\alpha \in \Gamma_{y}}(B(y, r) \cap \alpha Q) .
$$

Hence, we have

$$
B(y, r) \cap \gamma Q=\bigcup_{\alpha \in \Gamma_{y}}(B(y, r) \cap \alpha Q \cap \gamma Q) .
$$


Therefore $\gamma Q=\alpha Q$ for some $\alpha \in \Gamma_{y}$, since $\Gamma_{y}$ is finite. Hence $\alpha^{-1} \gamma Q=Q$ and $\alpha^{-1} \gamma x=y$. Thus $\alpha^{-1} \gamma \in \Sigma$ and $\pi_{\Gamma}$ induces an isometry from $Q / \Sigma$ to $\operatorname{Im}(\sigma)$.

We have a commutative diagram

$$
\begin{array}{ccc}
Q & \stackrel{\tilde{\eta}_{1}}{\longrightarrow} & E^{n} / V \\
\left(\pi_{\Gamma}\right)_{1} \downarrow & & \downarrow \pi_{\Gamma / \mathrm{N}} \\
\operatorname{Im}(\sigma) & \stackrel{\eta_{1}}{\longrightarrow}\left(E^{n} / V\right) /(\Gamma / \mathrm{N})
\end{array}
$$

with $\tilde{\eta}_{1}, \eta_{1},\left(\pi_{\Gamma}\right)_{1}$ the restrictions of $\tilde{\eta}, \eta, \pi_{\Gamma}$, respectively; moreover, $\tilde{\eta}_{1}$ and $\eta_{1}$ are homeomorphisms and the fibers of $\left(\pi_{\Gamma}\right)_{1}$ are the orbits of the action of $\Sigma$ on $Q$. Let $x \in Q$ such that $\pi_{\Gamma / \mathrm{N}} \tilde{\eta}_{1}(x)=\pi_{\Gamma / \mathrm{N}}(V+x)$ is an ordinary point of $\left(E^{n} / V\right) /(\Gamma / \mathrm{N})$, and let $\mathrm{N} \gamma \in \Gamma / \mathrm{N}$. Then there exists $\gamma^{\prime} \in \Sigma$ such that $\tilde{\eta}_{1}\left(\gamma^{\prime} x\right)=(\mathrm{N} \gamma) \tilde{\eta}_{1}(x)$. Hence $\left(\mathrm{N} \gamma^{\prime}\right)(V+x)=(\mathrm{N} \gamma)(V+x)$, and so $\mathrm{N} \gamma^{\prime}=\mathrm{N} \gamma$, since $\pi_{\Gamma / \mathrm{N}}(V+x)$ is an ordinary point of $\left(E^{n} / V\right) /(\Gamma / \mathrm{N})$. Therefore $\mathrm{N} \Sigma=\Gamma$. Hence, the space group extension

$$
1 \rightarrow \mathrm{N} \stackrel{i}{\longrightarrow} \Gamma \stackrel{p}{\longrightarrow} \Gamma / \mathrm{N} \rightarrow 1
$$

splits with $p$ mapping $\Sigma$ isometrically onto $\Gamma / \mathrm{N}$.

Theorem 17 Let $\mathrm{N}$ be a complete, torsion-free, normal subgroup of an $n$-dimensional space group $\Gamma$, and let $V=\operatorname{Span}(\mathrm{N})$. Let $\eta: E^{n} / \Gamma \rightarrow\left(E^{n} / V\right) /(\Gamma / \mathrm{N})$ be the fibration projection determined by $\mathrm{N}$. Then $\eta$ has an affine section if and only if the space group extension $1 \rightarrow \mathrm{N} \rightarrow \Gamma \rightarrow \Gamma / \mathrm{N} \rightarrow 1$ splits.

Proof If the space group extension $1 \rightarrow \mathrm{N} \rightarrow \Gamma \rightarrow \Gamma / \mathrm{N} \rightarrow 1$ splits, then $\eta$ has an affine section by Lemma 6 . If $\eta$ has an affine section $\sigma$, then $\operatorname{Im}(\sigma)$ intersects every generic fiber of $\eta$ at an ordinary point, since every point of a generic fiber is an ordinary point, because $\mathrm{N}$ is torsion-free. Therefore, the space group extension $1 \rightarrow \mathrm{N} \rightarrow \Gamma \rightarrow \Gamma / \mathrm{N} \rightarrow 1$ splits by Lemma 7 .

Lemma 8 Let $\mathrm{N}$ be a complete normal subgroup of an $n$-dimensional space group $\Gamma$, and let $V=\operatorname{Span}(\mathrm{N})$. Let $\eta: E^{n} / \Gamma \rightarrow\left(E^{n} / V\right) /(\Gamma / \mathrm{N})$ be the fibration projection determined by $\mathrm{N}$. If $V / \mathrm{N}$ has a point $\mathrm{N} v_{0}$ that is fixed by every isometry of $V / \mathrm{N}$, then the map $\sigma:\left(E^{n} / V\right) /(\Gamma / \mathrm{N}) \rightarrow E^{n} / \Gamma$, defined by $\sigma((\Gamma / \mathrm{N})(V+x))=\Gamma\left(v_{0}+x\right)$ for each $x \in V^{\perp}$, is a section of $\eta$ and an affine isometric embedding.

Proof Let $b+B \in \Gamma$. Write $b=c+d$ with $c \in V$ and $d \in V^{\perp}$. Then $(b+B) V=$ $V+d$. Let $a+A \in \mathrm{N}$ and let $v \in V$. Then we have

$$
(b+B)((a+A) v)=(b+B)(a+A)(b+B)^{-1}(b+B) v=\left(a^{\prime}+A^{\prime}\right)((b+B) v)
$$


with $a^{\prime}+A^{\prime} \in \mathrm{N}$, because $\mathrm{N}$ is a normal subgroup of $\Gamma$. Hence $b+B$ induces an isometry $(b+B)_{*}$ from $V / \mathrm{N}$ to $(V+d) / \mathrm{N}$ defined by $(b+B)_{*}(\mathrm{~N} v)=\mathrm{N}(b+B v)$. Now $(b+B)_{*}\left(\mathrm{~N} v_{0}\right)=\mathrm{N}\left(b+B v_{0}\right)$, and so $\mathrm{N}\left(b+B v_{0}\right)$ is fixed by every isometry of $(V+d) / \mathrm{N}$.

Next, we have $(d+I) V=V+d$ and $(d+I)((a+A) v)=(a+A)((d+I) v)$. Hence $d+I$ induces an isometry $(d+I)_{*}$ from $V / \mathrm{N}$ to $(V+d) / \mathrm{N}$ defined by $(d+I)_{*}(\mathrm{~N} v)=\mathrm{N}(v+d)$. Now $(d+I)_{*}\left(\mathrm{~N} v_{0}\right)=\mathrm{N}\left(v_{0}+d\right)$. Hence we have

$$
(d+I)_{*}(b+B)_{*}^{-1}\left(\mathrm{~N}\left(b+B v_{0}\right)\right)=\mathrm{N}\left(v_{0}+d\right),
$$

and so $\mathrm{N}\left(v_{0}+d\right)=\mathrm{N}\left(b+B v_{0}\right)$. Therefore there is an $a+A \in \mathrm{N}$ such that $(a+A)\left(b+B v_{0}\right)=v_{0}+d$. Hence $a+A c+A B v_{0}=v_{0}$.

The map $\tilde{\sigma}: E^{n} / V \rightarrow E^{n}$, defined by $\widetilde{\sigma}(V+x)=v_{0}+x$ for each $x \in V^{\perp}$, is an affine isometric embedding. Observe that

$$
\begin{aligned}
\tilde{\sigma}((b+B)(V+x)) & =\tilde{\sigma}(V+d+B x) \\
& =v_{0}+d+B x \\
& =a+A c+A B v_{0}+d+B x \\
& =a+A c+A B v_{0}+A d+A B x \\
& =(a+A b+A B)\left(v_{0}+x\right)=(a+A)(b+B) \tilde{\sigma}(V+x) .
\end{aligned}
$$

Hence $\tilde{\sigma}$ induces a map $\sigma:\left(E^{n} / V\right) /(\Gamma / \mathrm{N}) \rightarrow E^{n} / \Gamma$ defined by $\sigma((\Gamma / \mathrm{N})(V+x))=$ $\Gamma\left(v_{0}+x\right)$ for each $x \in V^{\perp}$. Now we have

$$
\eta \sigma((\Gamma / \mathrm{N})(V+x))=\eta\left(\Gamma\left(v_{0}+x\right)\right)=(\Gamma / \mathrm{N})(V+x),
$$

and so $\sigma$ is a section of $\eta$ and an affine isometric embedding.

Theorem 18 Let $\mathrm{N}$ be a complete normal subgroup of an $n$-dimensional space group $\Gamma$, and let $V=\operatorname{Span}(\mathrm{N})$. If $V / \mathrm{N}$ has an ordinary point $\mathrm{N} v_{0}$ that is fixed by every isometry of $V / \mathrm{N}$, then the space group extension $1 \rightarrow \mathrm{N} \rightarrow \Gamma \rightarrow \Gamma / \mathrm{N} \rightarrow 1$ splits.

Proof By conjugating $\Gamma$, we may assume that $\Gamma V / \Gamma$ is a generic fiber of $\eta$. Then $\Gamma V / \Gamma$ is isometric to $V / \mathrm{N}$. By Lemma 8 , the fibration projection $\eta: E^{n} / \Gamma \rightarrow$ $\left(E^{n} / V\right) /(\Gamma / \mathrm{N})$ has an affine section $\sigma$ such that $\operatorname{Im}(\sigma)$ intersects the fiber $\Gamma V / \Gamma$ in the ordinary point $\Gamma v_{0}$. Hence the space group extension $1 \rightarrow \mathrm{N} \rightarrow \Gamma \rightarrow \Gamma / \mathrm{N} \rightarrow 1$ splits by Lemma 7 . 
Example Consider a space group extension $1 \rightarrow \mathrm{N} \rightarrow \Gamma \rightarrow \Gamma / \mathrm{N} \rightarrow 1$ such that $\mathrm{N}$ is an infinite dihedral group. Then $V / \mathrm{N}$ is a closed interval. The midpoint of the closed interval $V / \mathrm{N}$ is an ordinary point of $V / \mathrm{N}$ that is fixed by both isometries of $V / \mathrm{N}$. Hence the space group extension $1 \rightarrow \mathrm{N} \rightarrow \Gamma \rightarrow \Gamma / \mathrm{N} \rightarrow 1$ splits by Theorem 18 .

Example We next consider an example that shows that the hypothesis that $\mathrm{N} v_{0}$ is an ordinary point cannot be dropped in Theorem 18. Let $\Gamma$ be the 3 -dimensional space group with IT number 113 in Table $1 \mathrm{~B}$ of [3]. Then $\Gamma=\left\langle t_{1}, t_{2}, t_{3}, \alpha, \beta, \gamma\right\rangle$ where $t_{i}=e_{i}+I$ for $i=1,2,3$ are the standard translations, and $\alpha=\frac{1}{2} e_{1}+\frac{1}{2} e_{2}+A$, $\beta=\frac{1}{2} e_{1}+B, \gamma=\frac{1}{2} e_{2}+C$, and

$$
A=\left(\begin{array}{ccc}
-1 & 0 & 0 \\
0 & -1 & 0 \\
0 & 0 & 1
\end{array}\right), \quad B=\left(\begin{array}{ccc}
0 & 1 & 0 \\
-1 & 0 & 0 \\
0 & 0 & -1
\end{array}\right), \quad C=\left(\begin{array}{ccc}
-1 & 0 & 0 \\
0 & 1 & 0 \\
0 & 0 & -1
\end{array}\right) \text {. }
$$

The group $\mathrm{N}=\left\langle t_{1}, t_{2}, \alpha, \beta \gamma\right\rangle$ is a complete normal subgroup of $\Gamma$ with $V=\operatorname{Span}(\mathrm{N})=$ Span $\left\{e_{1}, e_{2}\right\}$. The isomorphism type of $\mathrm{N}$ is $2 * 22$ in Conway's notation [6] or $\mathrm{cmm}$ in the international notation [16]. The flat orbifold $V / \mathrm{N}$ is a pointed hood. The orbifold $V / \mathrm{N}$ has a unique cone point $\mathrm{N}\left(\frac{1}{4} e_{1}+\frac{1}{4} e_{2}\right)$ which corresponds to the fixed point $\frac{1}{4} e_{1}+\frac{1}{4} e_{2}$ of the halfturn $\alpha$. Hence the cone point of $V / \mathrm{N}$ is fixed by every isometry of $V / \mathrm{N}$. Therefore the fibration projection $\eta: E^{3} / \Gamma \rightarrow\left(E^{3} / V\right) /(\Gamma / \mathrm{N})$ has an isometric section by Lemma 8 . However the space group extension $1 \rightarrow \mathrm{N} \rightarrow \Gamma \rightarrow \Gamma / \mathrm{N} \rightarrow 1$ does not split, since $\gamma$ projects to an element of order 2 in $\Gamma / \mathrm{N}$, but $((d+D) \gamma)^{2} \neq I$ for all $d+D$ in N. To see why, observe that there are only four possibilities for $D$, namely $D=I, A, B C, A B C$. Suppose $D=I$. Then $d=k e_{1}+\ell e_{2}$ for some integers $k$ and $\ell$, and we have

$$
((d+D) \gamma)^{2}=(1+2 \ell) e_{2}+I \neq I .
$$

The proofs of the other three cases for $D$ are similar. This example also shows that the hypothesis that $\mathrm{N}$ is torsion-free cannot be dropped in Theorem 17.

\section{Seifert fibrations}

We call a geometric fibration of a flat $n$-orbifold $M$ over a flat $(n-1)$-orbifold $B$ with generic fiber a connected, compact, flat $1-$ orbifold $F$ a geometric Seifert fibration. Here $F$ is either a circle or a closed interval. Seifert fibrations of compact flat 3orbifolds have been studied by Bonahon and Siebenmann [2], Dunbar [8] and Conway et al [7]. Every Seifert fibration of a compact flat 2- or 3-orbifold is isotopic to a geometric Seifert fibration by Proposition 2.12 of Boileau, Maillot and Porti [1] and the discussion in [2]. 
Let $F$ be a connected, compact, flat 1 -orbifold, and let $B$ be a flat $(n-1)$-orbifold $B$. Then $F \times B$ is a flat $n$-orbifold. The natural projection of $F \times B$ onto $B$ is a geometric Seifert fibration over $B$ with generic fiber $F$.

Let I be a closed interval, let $\widehat{B}$ be a connected, complete, flat $(n-1)$-orbifold, and let $\sigma$ be an involution of $\mathrm{I} \times \widehat{B}$ which acts diagonally, as a reflection on I and effectively and isometrically on $\widehat{B}$. Let $M=(\mathrm{I} \times \widehat{B}) /\langle\sigma\rangle$. Then $M$ is a flat $n$-orbifold and $B=\widehat{B} /\langle\sigma\rangle$ is a flat $(n-1)$-orbifold. The natural projection of $M$ onto $B$ is a geometric Seifert fibration over $B$ with generic fiber I. The flat orbifold $M$ is called the twisted I-bundle over $B$ determined by the orbifold double cover $\widehat{B}$ of $B$.

Theorem 19 Let $M$ be a connected, complete, flat $n$-orbifold, let $B$ be a flat $(n-1)$ orbifold, and let $\eta: M \rightarrow B$ be a geometric Seifert fibration with generic fiber a closed interval I. Let $\dot{\mathrm{I}}$ be the set of endpoints of I, let $\dot{M}$ be the union of all the endpoints of the fibers of $\eta$ that are determined by the endpoints of I, and let $\dot{\eta}: \dot{M} \rightarrow B$ be the restriction of $\eta$. Then $\dot{M}$ is a complete, flat $(n-1)$-orbifold, and $\dot{\eta}$ is a geometric fibration over $B$ with generic fiber the flat 0 -orbifold $\dot{\mathrm{I}}$. If $\dot{M}$ is disconnected, then $\eta$ is isometrically equivalent to the natural projection $\mathrm{I} \times B \rightarrow B$. If $\dot{M}$ is connected, then $\eta$ is isometrically equivalent to the natural projection of the twisted I-bundle over $B$ determined by the orbifold double covering $\dot{\eta}: \dot{M} \rightarrow B$.

Proof That $\dot{M}$ is a flat $(n-1)$-orbifold and $\dot{\eta}$ is a geometric fibration over $B$ with generic fiber $\dot{I}$ follows from the definition of the geometric fibration $\eta$. The orbifold $\dot{M}$ is complete, since $\dot{M}$ is a closed subspace of the complete metric space $M$. Let $B_{1 / 2}$ be the union of all the points of the fibers of $\eta$ that are determined by the midpoint of I. Then $B_{1 / 2}$ is a flat $(n-1)$-orbifold, and $\eta$ maps $B_{1 / 2}$ isometrically onto $B$. The geometric fibration $\dot{\eta}: \dot{M} \rightarrow B$ is geometrically equivalent to the fiberwise projection from $\dot{M}$ to $B_{1 / 2}$.

Suppose $\dot{M}$ is disconnected. Then $\dot{M}$ has exactly two connected components $B_{0}$ and $B_{1}$, and $\dot{\eta}$ maps $B_{i}$ isometrically onto $B$ for each $i$, since $\dot{\eta}: \dot{M} \rightarrow B$ is an orbifold double covering and $B$ is connected. Hence $B_{1 / 2}$ is two-sided in $M$. Parameterize I so that $\mathrm{I}=[0, \ell]$. Define $\phi: \mathrm{I} \times B \rightarrow M$ by $\phi(t, y)=y_{t}$ where $y_{t}$ is the point in $\eta^{-1}(y)$ which is at a distance $t$ from $B_{0}$ along $\eta^{-1}(y)$ towards $B_{1}$. Then $\phi$ is an isometry, and $\eta$ is isometrically equivalent to the natural projection $\mathrm{I} \times B \rightarrow B$.

Suppose $\dot{M}$ is connected. Then $B_{1 / 2}$ is one-sided in $M$. Let $\tau: \dot{M} \rightarrow \dot{M}$ be the continuous involution that transposes the endpoints of the fibers of $\eta$ that are isometric to I. Then $\tau$ is an isometry. Note that $x$ is fixed by $\tau$ if and only if $\eta^{-1}(\eta(x))$ is a singular fiber isometric to I folded in half. The geometric fibration $\dot{\eta}: \dot{M} \rightarrow B$ induces an isometry from $\dot{M} /\langle\tau\rangle$ to $B$. Define $\phi: \mathrm{I} \times \dot{M} \rightarrow M$ by $\phi(t, x)=x_{t}$ where $x_{t}$ is 
the point of $\eta^{-1}(\eta(x))$ which is at a distance $t$ from $x$ if $t \leq \ell / 2$ or at a distance $\ell-t$ from $\tau(x)$ if $t \geq \ell / 2$. Let $\sigma: \mathrm{I} \times \dot{M} \rightarrow \mathrm{I} \times \dot{M}$ be the isometry that acts diagonally, as the reflection in I, and by $\tau$ on $\dot{M}$. Then $\phi$ induces an isometry from $(\mathrm{I} \times \dot{M}) /\langle\sigma\rangle$ to $M$, and $\eta$ is isometrically equivalent to the natural projection of the twisted I-bundle over $B$ determined by the orbifold double covering $\dot{\eta}: \dot{M} \rightarrow B$.

Theorem 20 Suppose that

$$
1 \rightarrow \mathrm{N} \stackrel{i}{\rightarrow} \Gamma \stackrel{p}{\rightarrow} \Gamma / \mathrm{N} \rightarrow 1
$$

is a space group extension such that $\mathrm{N}$ is an infinite dihedral group, then $\Gamma$ has a subgroup $\Sigma$ such that $p$ maps $\Sigma$ isomorphically onto $\Gamma / \mathrm{N}$, and either $\Gamma=\mathrm{N} \times \Sigma$ and $\Sigma$ is unique and orthogonal to $\mathrm{N}$, or else $\Sigma$ has a subgroup $\Sigma_{0}$ of index 2 such that if $\Gamma_{0}=\mathrm{N} \Sigma_{0}$. Then $\Gamma_{0}=\mathrm{N} \times \Sigma_{0}$ and $\Sigma_{0}$ is unique, but $\Sigma$ is not necessarily unique; moreover $\Sigma_{0}$ is a complete normal subgroup of $\Gamma$, which is orthogonal to $\mathrm{N}$, and $\Gamma / \Sigma_{0}$ is an infinite dihedral group.

Proof Let $V=\operatorname{Span}(\mathrm{N})$, and let $n=\operatorname{dim}(\Gamma)$. Then $\mathrm{I}=V / \mathrm{N}$ is a closed interval and the fibration projection $\eta: E^{n} / \Gamma \rightarrow\left(E^{n} / V\right) /(\Gamma / \mathrm{N})$ is a geometric Seifert fibration with generic fiber I. Let $M=E^{n} / \Gamma$. By Theorem 18, the group $\Gamma$ has a subgroup $\Sigma$ such that $p$ maps $\Sigma$ isomorphically onto $\Gamma / \mathrm{N}$, and by Theorem 19, either $\Gamma=\mathrm{N} \times \Sigma$, with $\Sigma$ orthogonal to $\mathrm{N}$, if $\dot{M}$ is disconnected, or else $\Sigma$ has a subgroup $\Sigma_{0}$ of index 2, if $\dot{M}$ is connected, corresponding to the orbifold double cover $\dot{M}$ of $\left(E^{n} / V\right) /(\Gamma / \mathrm{N})$, such that if $\Gamma_{0}=\mathrm{N} \Sigma_{0}$, then $\Gamma_{0}=\mathrm{N} \times \Sigma_{0}$, since $\mathrm{I} \times \dot{M}$ double covers $M$. If $\Gamma=\mathrm{N} \times \Sigma$, then $\Sigma$ is unique, since $\Sigma$ is the centralizer of $\mathrm{N}$ in $\Gamma$. If $\Gamma \neq \mathrm{N} \times \Sigma$, then $\Sigma_{0}$ is unique, since $\Sigma_{0}$ is the centralizer of $\mathrm{N}$ in $\Gamma$. To see that $\Sigma$ is not necessarily unique, see example (5) in the next section. The group $\Sigma_{0}$ is normal in $\Gamma$, since $\Sigma_{0}$ is the centralizer of a normal subgroup of $\Gamma$. The group $\Sigma_{0}$ is complete and $\Gamma / \Sigma_{0}$ is an infinite dihedral group, since $\Sigma_{0}$ corresponds to the generic fiber of the geometric fibration of $(\mathrm{I} \times \dot{M}) /\langle\sigma\rangle$ over $\mathrm{I} /\langle\sigma\rangle$ induced by projection along the first factor. Finally $\Sigma_{0}$ is orthogonal to $\mathrm{N}$, since $\Sigma$ can be chosen to be orthogonal to $\mathrm{N}$ as in the proof of Lemma 8.

\section{Reducible 2-dimensional crystallographic groups}

Let $\Gamma$ be a 2-dimensional space group. Then every nontrivial geometric fibration of $E^{2} / \Gamma$ is a Seifert fibration. If $b+B \in \Gamma$ and $B$ has no 1 -dimensional invariant vector space, then $E^{2} / \Gamma$ does not Seifert fiber. Hence if $B$ is a rotation of order 3 or 4 , then $E^{2} / \Gamma$ does not Seifert fiber. This excludes 8 of the 2-dimensional space 
group isomorphism types. The orbifolds of the remaining 9 isomorphism types do Seifert fiber. We now describe all of these geometric Seifert fibrations. We denote a circle by $\mathrm{O}$ and a closed interval by $\mathrm{I}$.

We consider the groups in the order of Table 1A of Brown et al [3] which corresponds to the IT order [10]. We use the generators for the representatives of the isomorphism types of the 2-dimensional space groups listed in Table 1A of [3]. Let $t_{1}=e_{1}+I$ and $t_{2}=e_{2}+I$ be the standard basis translations, and let

$$
A=\left(\begin{array}{cc}
-1 & 0 \\
0 & -1
\end{array}\right), \quad B=\left(\begin{array}{cc}
1 & 0 \\
0 & -1
\end{array}\right), \quad C=\left(\begin{array}{ll}
0 & 1 \\
1 & 0
\end{array}\right) .
$$

Let $a, b$ be relatively prime integers, let $c, d$ be integers such that $a d-b c=1$, and let $\phi: E^{2} \rightarrow E^{2}$ be the linear automorphism defined by $\phi\left(e_{1}\right)=(a, b)$ and $\phi\left(e_{2}\right)=(c, d)$. The symbol $\rtimes$ denotes a semidirect product of groups.

(1) Let $\Gamma=\left\langle t_{1}, t_{2}\right\rangle$. The isomorphism type of $\Gamma$ is $\circ(p 1)$. Here $\circ$ is Conway's notation [6] and $p 1$ is the IT notation [16]. The orbifold $E^{2} / \Gamma$ is a flat torus and $E^{2} / \Gamma$ is the cartesian product $\mathrm{O} \times \mathrm{O}$. The proper, complete, normal subgroups of $\Gamma$ are of the form $\left\langle t_{1}^{a} t_{2}^{b}\right\rangle$. We have $\Gamma=\left\langle t_{1}^{a} t_{2}^{b}\right\rangle \times\left\langle t_{1}^{c} t_{2}^{d}\right\rangle$, and so $E^{2} / \Gamma$ is a geometric trivial fiber bundle over $\mathrm{O}$, with fiber $\mathrm{O}$, in infinitely many ways. The linear automorphism $\phi$ normalizes $\Gamma$ and conjugates $\left\langle t_{1}\right\rangle$ to $\left\langle t_{1}^{a} t_{2}^{b}\right\rangle$. Therefore, all the geometric Seifert fiberings of $E^{2} / \Gamma$ are affinely equivalent.

(2) Let $\Gamma=\left\langle t_{1}, t_{2}, A\right\rangle$. The isomorphism type of $\Gamma$ is $2222(p 2)$ and $E^{2} / \Gamma$ is a flat pillow. The proper, complete, normal subgroups of $\Gamma$ are of the form $\left\langle t_{1}^{a} t_{2}^{b}\right\rangle$. We have $\Gamma=\left\langle t_{1}^{a} t_{2}^{b}\right\rangle \rtimes\left\langle t_{1}^{c} t_{2}^{d}, A\right\rangle$, and so $E^{2} / \Gamma$ geometrically fibers over I, with generic fiber O, in infinitely many ways. The linear automorphism $\phi$ normalizes $\Gamma$ and conjugates $\left\langle t_{1}\right\rangle$ to $\left\langle t_{1}^{a} t_{2}^{b}\right\rangle$. Therefore, all the geometric Seifert fiberings of $E^{2} / \Gamma$ are affinely equivalent.

(3) Let $\Gamma=\left\langle t_{1}, t_{2}, B\right\rangle$. The isomorphism type of $\Gamma$ is $* *(p m)$ and $E^{2} / \Gamma$ is a flat annulus. The proper, complete, normal subgroups of $\Gamma$ are $Z(\Gamma)=\left\langle t_{1}\right\rangle$ and $\left\langle t_{2}, B\right\rangle$. We have $\Gamma=\left\langle t_{1}\right\rangle \times\left\langle t_{2}, B\right\rangle$, and so $E^{2} / \Gamma$ is the cartesian product $\mathrm{O} \times \mathrm{I}$.

(4) Let $\Gamma=\left\langle t_{1}, t_{2}, \beta\right\rangle$ where $\beta=\frac{1}{2} e_{1}+B$. The isomorphism type of $\Gamma$ is $\times \times(p g)$ and $E^{2} / \Gamma$ is a flat Klein bottle. The proper, complete, normal subgroups of $\Gamma$ are $Z(\Gamma)=\left\langle t_{1}\right\rangle$ and $\left\langle t_{2}\right\rangle$. Now $\Gamma /\left\langle t_{1}\right\rangle \cong D_{\infty}$, and so $E^{2} / \Gamma$ geometrically fibers over I with generic fiber $\mathrm{O}$. The extension $1 \rightarrow\left\langle t_{1}\right\rangle \rightarrow \Gamma \rightarrow D_{\infty} \rightarrow 1$ does not split, since $\Gamma$ is torsion-free. Also $\Gamma=\left\langle t_{2}\right\rangle \rtimes\langle\beta\rangle$, and so $E^{2} / \Gamma$ is a geometric fiber bundle over $\mathrm{O}$ with fiber $\mathrm{O}$.

(5) Let $\Gamma=\left\langle t_{1}, t_{2}, C\right\rangle$. The isomorphism type of $\Gamma$ is $* \times(\mathrm{cm})$ and $E^{2} / \Gamma$ is a flat Möbius band. The proper, complete, normal subgroups of $\Gamma$ are $Z(\Gamma)=\left\langle t_{1} t_{2}\right\rangle$ 
and $\left\langle t_{1} t_{2}^{-1}, C\right\rangle$. Now $\Gamma /\left\langle t_{1} t_{2}\right\rangle \cong D_{\infty}$, and so $E^{2} / \Gamma$ geometrically fibers over I with generic fiber $\mathrm{O}$. The extension $1 \rightarrow\left\langle t_{1} t_{2}\right\rangle \rightarrow \Gamma \rightarrow D_{\infty} \rightarrow 1$ does not split, since $\Gamma$ is not the direct product of $\left\langle t_{1} t_{2}\right\rangle$ and $D_{\infty}$. Also $\Gamma=\left\langle t_{1} t_{2}^{-1}, C\right\rangle \rtimes\left\langle t_{i} C\right\rangle$ for $i=1,2$, and so $E^{2} / \Gamma$ is a geometric fiber bundle over $\mathrm{O}$ with fiber I. We have $\left(t_{i} C\right)^{2}=t_{1} t_{2}$ for $i=1,2$, and $\left\langle t_{1} t_{2}\right\rangle$ is the centralizer of $\left\langle t_{1} t_{2}^{-1}, C\right\rangle$ in $\Gamma$. As $\left(t_{1} C\right)^{-1}=t_{2}^{-1} C$, we have that $\left\langle t_{1} C\right\rangle \neq\left\langle t_{2} C\right\rangle$. Hence the subgroup $\Sigma=\left\langle t_{1} C\right\rangle$ in Theorem 20 is not necessarily unique.

(6) Let $\Gamma=\left\langle t_{1}, t_{2}, A, B\right\rangle$. The isomorphism type of $\Gamma$ is $* 2222(\mathrm{pmm})$ and $E^{2} / \Gamma$ is a square. The proper, complete, normal subgroups of $\Gamma$ are $\left\langle t_{1}, A B\right\rangle$ and $\left\langle t_{2}, B\right\rangle$. Now $\Gamma=\left\langle t_{1}, A B\right\rangle \times\left\langle t_{2}, B\right\rangle$, and so $E^{2} / \Gamma$ is the cartesian product $\mathrm{I} \times \mathrm{I}$. The linear automorphism $\sigma: E^{2} \rightarrow E^{2}$, defined by $\sigma\left(e_{1}\right)=e_{2}$ and $\sigma\left(e_{2}\right)=e_{1}$, normalizes $\Gamma$ and conjugates $\left\langle t_{1}, A B\right\rangle$ to $\left\langle t_{2}, B\right\rangle$. Therefore, the two geometric Seifert fiberings of $E^{2} / \Gamma$ are isometrically equivalent.

(7) Let $\Gamma=\left\langle t_{1}, t_{2}, A, \beta\right\rangle$ where $\beta=\frac{1}{2} e_{2}+B$. The isomorphism type is $22 *(p m g)$ and $E^{2} / \Gamma$ is a pillowcase. The proper, complete, normal subgroups of $\Gamma$ are $\left\langle t_{1}\right\rangle$ and $\left\langle t_{2}, \beta\right\rangle$. Now $\Gamma=\left\langle t_{1}\right\rangle \rtimes\langle A, \beta\rangle$, and so $E^{2} / \Gamma$ geometrically fibers over I with generic fiber O. Also $\Gamma=\left\langle t_{2}, \beta\right\rangle \rtimes\left\langle t_{1}, A\right\rangle$, and so $E^{2} / \Gamma$ geometrically fibers over I with generic fiber I.

(8) Let $\Gamma=\left\langle t_{1}, t_{2}, A, \beta\right\rangle$ where $\beta=\frac{1}{2} e_{1}+\frac{1}{2} e_{2}+B$. The isomorphism type of $\Gamma$ is $22 \times(\mathrm{pgg})$ and $E^{2} / \Gamma$ is a projective pillow. The proper, complete, normal subgroups of $\Gamma$ are $\left\langle t_{1}\right\rangle$ and $\left\langle t_{2}\right\rangle$. Now $\Gamma /\left\langle t_{i}\right\rangle \cong D_{\infty}$ for $i=1,2$, and so $E^{2} / \Gamma$ geometrically fibers over I, with generic fiber $\mathrm{O}$, in two ways. The extension $1 \rightarrow\left\langle t_{1}\right\rangle \rightarrow \Gamma \rightarrow$ $D_{\infty} \rightarrow 1$ does not split, since $\beta$ projects to an element of order 2 but $t_{1}^{k} \beta$ is a glidereflection for each integer $k$. The linear automorphism $\sigma: E^{2} \rightarrow E^{2}$, defined by $\sigma\left(e_{1}\right)=e_{2}$ and $\sigma\left(e_{2}\right)=e_{1}$, normalizes $\Gamma$ and conjugates $\left\langle t_{1}\right\rangle$ to $\left\langle t_{2}\right\rangle$. Therefore, the two geometric Seifert fiberings of $E^{2} / \Gamma$ are isometrically equivalent. Hence the extension $1 \rightarrow\left\langle t_{2}\right\rangle \rightarrow \Gamma \rightarrow D_{\infty} \rightarrow 1$ also does not split.

(9) Let $\Gamma=\left\langle t_{1}, t_{2}, A, C\right\rangle$. The isomorphism type of $\Gamma$ is $2 * 22(\mathrm{cmm})$ and $E^{2} / \Gamma$ is a pointed hood. The proper, complete, normal subgroups of $\Gamma$ are $\left\langle t_{1} t_{2}, A C\right\rangle$ and $\left\langle t_{1} t_{2}^{-1}, C\right\rangle$. Now $\Gamma=\left\langle t_{1} t_{2}, A C\right\rangle \rtimes\left\langle t_{1} A, C\right\rangle$ and $\Gamma=\left\langle t_{1} t_{2}^{-1}, C\right\rangle \rtimes\left\langle t_{1} A, A C\right\rangle$, and so $E^{2} / \Gamma$ geometrically fibers over I, with generic fiber I, in two ways. The linear automorphism $\sigma: E^{2} \rightarrow E^{2}$, defined by $\sigma\left(e_{1}\right)=e_{1}$ and $\sigma\left(e_{2}\right)=-e_{2}$, normalizes $\Gamma$ and conjugates $\left\langle t_{1} t_{2}, A C\right\rangle$ to $\left\langle t_{1} t_{2}^{-1}, C\right\rangle$. Therefore, the two geometric Seifert fiberings of $E^{2} / \Gamma$ are isometrically equivalent. 


\section{Co-Seifert fibrations}

Let $M$ be a flat $n$-orbifold. We call a geometric fibration of $M$ over a connected, compact, flat 1 -orbifold $B$, with generic fiber a flat $(n-1)$-orbifold $F$, a geometric co-Seifert fibration. Here $B$ is either a circle $\mathrm{O}$ or a closed interval $\mathrm{I}$. The structure of a geometric co-Seifert fibration $\eta: M \rightarrow B$ tells you a lot about the geometry and topology of the orbifold $M$. If the base $B$ is a circle $\mathrm{O}$, then $M$ is a geometric fiber bundle over $\mathrm{O}$ with fiber $F$. Hence $M$ is a mapping torus over $F$ with monodromy an isometry of $F$. Moreover, the singular set $\Sigma$ of $M$ is a fiber bundle over $\mathrm{O}$ with fiber the singular set $\Sigma_{*}$ of $F$.

Example Take $\Gamma$ to be as in (3) in Section 10. Then $M=E^{2} / \Gamma$ is a flat annulus, $M$ is a geometric fiber bundle over $\mathrm{O}$ with fiber $\mathrm{I}$, and $M$ is the mapping torus over I with monodromy the identity map of I. The singular set $\Sigma$ of $M$ is the boundary of the annulus which is a trivial fiber bundle over $\mathrm{O}$ with fiber the endpoints of $\mathrm{I}$.

Example Take $\Gamma$ to be as in (5) in Section 10. Then $M=E^{2} / \Gamma$ is a flat Möbius band, $M$ is a geometric fiber bundle over $\mathrm{O}$ with fiber $\mathrm{I}$, and $M$ is the mapping torus over I with monodromy the reflection of I about the midpoint of I. The singular set $\Sigma$ of $M$ is the boundary of the Möbius band, which is a nontrivial fiber bundle over $\mathrm{O}$ with fiber the endpoints of I.

Now suppose the base $B$ is a closed interval $\mathrm{I}$ with endpoints 0 and 1 . Let $\mathrm{I}^{\circ}$ be the open interval $(0,1)$. Then $\eta^{-1}\left(\mathrm{I}^{\circ}\right)$ is the cartesian product of $F$ and $\mathrm{I}^{\circ}$. Hence $M$ is obtained from the open tube $\eta^{-1}\left(\mathrm{I}^{\circ}\right)$ by adjoining the two singular fibers $F_{0}=\eta^{-1}(0)$ and $F_{1}=\eta^{-1}(1)$ of $\eta$ to the ends of the tube, one to each end. Let $G_{0}$ and $G_{1}$ be the finite groups of order 2 in the definition of the geometric fibration $\eta$ such that $F_{i}$ is isometric to $F / G_{i}$ for $i=0,1$. There are three cases to consider.

The first case is when $G_{0}$ and $G_{1}$ both act trivially on $F$. Then $M$ is the cartesian product $F \times \mathrm{I}$. The singular set $\Sigma$ of $M$ is $F_{0} \cup\left(\Sigma_{*} \times \mathrm{I}\right) \cup F_{1}$ where $\Sigma_{*}$ is the singular set of $F$.

Example Take $\Gamma$ to be as in (6) in Section 10. Then $M=E^{2} / \Gamma$ is a square and $M$ is the cartesian product $\mathrm{I} \times \mathrm{I}$. The singular set of $M$ is the boundary of the square, which is the union of the 4 closed intervals $F_{0}, \Sigma_{*} \times \mathrm{I}, F_{1}$.

The second case is when one of $G_{0}$ and $G_{1}$ acts trivially on $F$ and the other does not, say $G_{1}$ acts trivially on $F$ and $G_{0}$ does not. Then $M$ is isometric to $(F \times[-1,1]) / G_{0}$ where $G_{0}$ acts diagonally, isometrically on $F$, and orthogonally on $[-1,1]$ by reflecting $[-1,1]$ about 0 ; in other words, $M$ is a twisted I-bundle over $F_{0}$ with monodromy determined by the action of $G_{0}$ on $F$. The singular set $\Sigma$ of $M$ is $\Sigma_{0} \cup\left(\Sigma_{*} \times(0,1]\right) \cup F_{1}$ where $\Sigma_{0}$ is the singular set of $F_{0}$. 
Example Take $\Gamma$ to be as in (7) in Section 10. Then $M=E^{2} / \Gamma$ is a pillowcase and $M$ geometrically fibers over I with generic fiber $\mathrm{O}$ as in the second case. We have that $M$ is a twisted I-bundle over I with monodromy determined by a reflection of $\mathrm{O}$. The singular set of $M$ is the union of the boundary circle $F_{1}$ of $M$ together with the two cone points which form the singular set of the closed interval $F_{0}$.

Example Take $\Gamma$ to be as in (9) in Section 10. Then $M=E^{2} / \Gamma$ is a pointed hood and $M$ geometrically fibers over I with generic fiber I as in the second case. We have that $M$ is a twisted I-bundle over I with monodromy determined by the reflection of I. The singular set $\Sigma$ of $M$ is the boundary of $M$ together with a single cone point. Observe that $\Sigma$ is the union of the closed interval $F_{1}$, two half-open intervals $\Sigma_{*} \times(0,1]$, and the end points of $F_{0}$, one of which is the cone point and the other joins the two half-open intervals $\Sigma_{*} \times(0,1]$ to form a closed interval.

The third case is when $G_{0}$ and $G_{1}$ both act nontrivially on $F$. Let $M_{0}=\eta^{-1}([0,1 / 2])$ and $M_{1}=\eta^{-1}([1 / 2,1])$, and let $F_{1 / 2}=\eta^{-1}(1 / 2)$. Then $M_{i}$ is a twisted I-bundle over $F_{i}$ with monodromy determined by the action of $G_{i}$ on $F$ for $i=0,1$. Note that $M_{i}$ is a flat $n$-orbifold with totally geodesic boundary $F_{1 / 2}$ for each $i=0,1$. We have that $M=M_{0} \cup M_{1}$ with $M_{0} \cap M_{1}=F_{1 / 2}$. The singular set $\Sigma$ of $M$ is $\Sigma_{0} \cup\left(\Sigma_{*} \times(0,1)\right) \cup \Sigma_{1}$ where $\Sigma_{i}$ is the singular set of $F_{i}$ for $i=0,1$.

Example Take $\Gamma$ to be as in (2) in Section 10. Then $M=E^{2} / \Gamma$ is a pillow and $M$ geometrically fibers over I with generic fiber $\mathrm{O}$ as in the third case. Observe that $M_{i}$ is a pillowcase for each $i=0,1$, with $F_{1 / 2}$ their common boundary circle. The singular set of $M$ is four cone points which form the union of the endpoints of the closed intervals $F_{0}$ and $F_{1}$.

Example Take $\Gamma$ to be as in (8) in Section 10. Then $M=E^{2} / \Gamma$ is a projective pillow and $M$ geometrically fibers over I with generic fiber $\mathrm{O}$ as in the third case. Here $G_{0}$ acts be a reflection on $\mathrm{O}$ and $G_{1}$ acts by the a half-turn on $\mathrm{O}$. Therefore $M_{0}$ is a pillowcase and $M_{1}$ is a Möbius band, with $F_{1 / 2}$ is their common boundary circle. Therefore $M$ is topologically a projective plane. The singular set of $M$ consists of two cone points which are the endpoints of the closed interval $F_{0}$.

Example Take $\Gamma$ to be as in (7) in Section 10. Then $M=E^{2} / \Gamma$ is a pillowcase and $M$ geometrically fibers over I with generic fiber I as in the second case. Here $G_{i}$ acts on I by reflection for $i=0,1, M_{i}$ is a pointed hood for each $i=0,1$, and $F_{1 / 2}$ is a closed interval which is half the boundary of each hood. The singular set consists of the endpoints of the closed intervals $F_{0}$ and $F_{1}$ and two open intervals $\Sigma_{*} \times(0,1)$. 
The two open intervals $\Sigma_{*} \times(0,1)$ together with one endpoint from each of $F_{0}$ and $F_{1}$ form the boundary circle of $M$.

Let $\Gamma$ be a 3-dimensional, orientation preserving, space group. In [8], Dunbar describes the topology and geometry of the flat orbifold $E^{3} / \Gamma$. When $E^{3} / \Gamma$ co-Seifert fibers, the structure of a co-Seifert fibration can effectively be used to determine the topology and geometry of the orbifold $E^{3} / \Gamma$.

\section{Reducible 3-dimensional crystallographic groups}

Let $\Gamma$ be a 3 -dimensional space group with point group $\Pi$. Suppose $E^{3} / \Gamma$ geometrically fibers over a 1 - or 2-dimensional flat orbifold. By Theorem 7 , the geometric fibration of $E^{3} / \Gamma$ is determined by a complete normal subgroup $\mathrm{N}$ of $\Gamma$. Let $V=\operatorname{Span}(\mathrm{N})$. Then $V$ is either a $1-$ or $2-$ dimensional vector subspace of $E^{3}$. As both $V$ and $V^{\perp}$ are invariant by $\Pi$, the group $\Pi$ has a 1 -dimensional invariant vector space.

The group $\Gamma$ is said to be reducible or irreducible according as its point group $\Pi$ has or has not a 1-dimensional invariant vector space. It is well known to crystallographers that reducibility of 3 -dimensional space groups is equivalent to $\mathbb{Z}$-reducibility. Hence $E^{3} / \Gamma$ geometrically fibers over a flat $1-$ or 2 -orbifold if and only if $\Gamma$ is reducible by Theorems 4,7 and 11 .

There are six families of 3-dimensional space group isomorphism types, the triclinic, monoclinic, orthorhombic, tetragonal, hexagonal, and cubic families. See Table 1B of Brown et al [3] where the six families are listed in the above order. The cubic family consists of all the irreducible isomorphism types. There are 35 irreducible 3-dimensional space group isomorphism types and 184 reducible 3-dimensional space group isomorphism types.

All the geometric Seifert fibrations of $E^{3} / \Gamma$, up to affine equivalence, were neatly described by Conway et al in their 2001 paper [7]. We will describe the Conway et al formulation of a geometric fibration of $E^{3} / \Gamma$ over a flat 2-orbifold and show how it determines a dual geometric fibration of $E^{3} / \Gamma$ over a flat 1 -orbifold with the same invariant 1 -dimensional vector space.

Let $\Gamma$ is a reducible 3-dimensional space group. Conway et al represent a geometric fibration of $E^{3} / \Gamma$ over a flat 2-orbifold by an exact sequence

$$
1 \rightarrow \mathrm{K} \longrightarrow \Gamma \stackrel{\pi}{\longrightarrow} \mathrm{H} \rightarrow 1
$$

where $\mathrm{H}$ is a 2 -dimensional space group and $\mathrm{K}=\operatorname{ker}(\pi)$. Here an element $g$ of $\Gamma$ is represented in the form $\left(g_{H}, g_{V}\right)$ where $\pi(g)=g_{H}$ and $g_{V}$ is the third coordinate 
function of $g$. The group $\mathrm{K}$ is generated by either the translation $e_{3}+I$ or $e_{3}+I$ and the reflection $A=\operatorname{diag}(1,1,-1)$. The group extension is specified by normalized choices of the third coordinate functions for a set of generators of $\mathrm{H}$. The choices are such that $\left\{\left(I, g_{V}\right): g \in \Gamma\right\}$ is a discrete group $\Lambda$ of isometries of $E^{3}$ containing $\mathrm{K}$ as a normal subgroup of finite index. The projection $\phi: g \mapsto g_{V}$ is a group homomorphism whose image is a 1 -dimensional space group $\Lambda_{1}$ isomorphic to $\Lambda$ via the mapping $g_{V} \mapsto\left(I, g_{V}\right)$. Let $\mathrm{N}=\operatorname{ker}(\phi)$. Then $\mathrm{N}$ is complete by Theorem 5 . Hence $E^{3} / \Gamma$ geometrically fibers over a flat 1 -orbifold corresponding to the group extension

$$
1 \rightarrow \mathrm{N} \longrightarrow \Gamma \stackrel{\phi}{\longrightarrow} \Lambda_{1} \rightarrow 1
$$

by Theorem 4 with $\operatorname{Span}(\mathrm{N})=\operatorname{Span}\left\{e_{1}, e_{2}\right\}$. Hence $\mathrm{K}$ and $\mathrm{N}$ are orthogonal. We call $\mathrm{N}$ the orthogonal dual of $\mathrm{K}$, and we say that $\mathrm{K}$ and $\mathrm{N}$ correspond under orthogonal duality.

As $\mathrm{K} \cap \mathrm{N}=\{I\}$, we have that $\pi$ maps $\mathrm{N}$ isomorphically onto a normal subgroup of $\mathrm{H}$, moreover

$$
\begin{aligned}
\mathrm{H} / \pi(\mathrm{N}) & \cong(\Gamma / \mathrm{K}) /(\mathrm{KN} / \mathrm{K}) \\
& \cong \Gamma / \mathrm{KN} \\
& \cong(\Gamma / \mathrm{N}) /(\mathrm{KN} / \mathrm{N}) \\
& \cong \Lambda_{1} / \phi(\mathrm{K}) \cong \Lambda / \mathrm{K} .
\end{aligned}
$$

All the elements of $\mathrm{K}$ commute with all the elements of $\mathrm{N}$. The group $\Gamma$ is the direct product of $\mathrm{K}$ and $\mathrm{N}$ if and only if $\mathrm{K}=\Lambda$. This corresponds to the occurrence of a row of $0+\cdots 0+0-$ (resp. $0+\cdots 0+$ ) in the couplings column of Table 1 of [7] for interval (resp. circular) fibrations.

The group $\Lambda$ is infinite cyclic if and only if all the elements of $\Lambda$ are translations. This corresponds to the occurrence of a row of all plus signs in the couplings column of Table 1 of [7].

The tetragonal and hexagonal families of 3-dimensional space group isomorphism types (IT numbers 75-194) consist of all the reducible group isomorphism types whose point group has a unique 1 -dimensional invariant vector space. If $\Gamma$ belongs to one of these 110 isomorphism types, then $\Gamma$ has a unique 1 -dimensional, complete, normal subgroup $\mathrm{K}$ and a unique 2-dimensional, complete, normal subgroup $\mathrm{N}$; moreover $\mathrm{K}$ and $\mathrm{N}$ are orthogonal. This is the case if and only if in the Conway et al representation of $\Gamma$ the group $\mathrm{H}$ is irreducible. Hence, the classification of the geometric co-Seifert fibrations of $E^{3} / \Gamma$ corresponds via orthogonal duality to the Conway et al classification of the geometric Seifert fibrations of $E^{3} / \Gamma$ when $\Gamma$ belongs to the tetragonal or hexagonal families. 
The orthorhombic family (IT numbers 16-74) consists of all the reducible group isomorphism types whose point group has exactly three orthogonal 1-dimensional invariant vector spaces. If $\Gamma$ belongs to one of these 59 isomorphism types, then $\Gamma$ has exactly three 1-dimensional, complete, normal subgroups $\mathrm{K}_{1}, \mathrm{~K}_{2}, \mathrm{~K}_{3}$, and $\Gamma$ has exactly three 2-dimensional, complete, normal subgroups $\mathrm{N}_{1}, \mathrm{~N}_{2}, \mathrm{~N}_{3}$. This is the case if and only if in the Conway et al representation of $\Gamma$ the group $\mathrm{H}$ has exactly two proper complete, normal subgroups. We order $\mathrm{K}_{i}$ and $\mathrm{N}_{i}$ so that $V_{i}=\operatorname{Span}\left(\mathrm{K}_{i}\right)$ and $W_{i}=\operatorname{Span}\left(\mathrm{N}_{i}\right)$ are orthogonal complements for each $i$. The 1 -dimensional vector spaces $V_{1}, V_{2}, V_{3}$ are mutually orthogonal.

Suppose $\alpha: E^{3} \rightarrow E^{3}$ is an affine homeomorphism such that $\alpha \Gamma \alpha^{-1}=\Gamma^{\prime}$ with $\Gamma^{\prime}$ a space group. Define $\mathrm{K}_{i}^{\prime}=\alpha \mathrm{K}_{i} \alpha^{-1}$ and $\mathrm{N}_{i}^{\prime}=\left(\mathrm{K}_{i}^{\prime}\right)^{\perp}$ for $i=1,2,3$. Let $V_{i}^{\prime}=\operatorname{Span}\left(\mathrm{K}_{i}^{\prime}\right)$ and $W_{i}^{\prime}=\operatorname{Span}\left(\mathrm{N}_{i}^{\prime}\right)$ for each $i$. Let $\alpha=a+A$ with $A$ a linear automorphism of $E^{3}$. Then $A V_{i}=V_{i}^{\prime}$ for each $i$. Now $\alpha \mathrm{N}_{i} \alpha^{-1}=\mathrm{N}_{j}^{\prime}$ for some $j$ by Corollary 2 . Therefore $A W_{i}=W_{j}^{\prime}$. As $A V_{i} \cap A W_{i}=\{0\}$, we must have that $j=i$ for each $i=1,2,3$. Likewise if $\mathrm{N}_{i}^{\prime}=\alpha \mathrm{N}_{i} \alpha^{-1}$ and $\mathrm{K}_{i}^{\prime}=\left(\mathrm{N}_{i}^{\prime}\right)^{\perp}$ for each $i=1,2,3$, then $\alpha\left(\mathrm{K}_{i}\right) \alpha^{-1}=\mathrm{K}_{i}^{\prime}$ for each $i=1,2,3$. Thus if $\mathrm{N}$ is a complete normal subgroup of $\Gamma$, then $\alpha\left(\mathrm{N}^{\perp}\right) \alpha^{-1}=\left(\alpha \mathrm{N} \alpha^{-1}\right)^{\perp}$. By Theorem 10, the geometric fibrations of $E^{3} / \Gamma$ and $E^{3} / \Gamma^{\prime}$ determined by complete normal subgroups $\mathrm{N}$ of $\Gamma$ and $\mathrm{N}^{\prime}$ of $\Gamma^{\prime}$ are affinely equivalent if and only if the geometric fibrations of $E^{3} / \Gamma$ and $E^{3} / \Gamma^{\prime}$ determined by $\mathrm{N}^{\perp}$ and $\left(\mathrm{N}^{\prime}\right)^{\perp}$ are affinely equivalent. Hence, the classification of the geometric co-Seifert fibrations of $E^{3} / \Gamma$ corresponds via orthogonal duality to the Conway et al classification of the geometric Seifert fibrations of $E^{3} / \Gamma$ when $\Gamma$ belongs to the orthorhombic family.

The triclinic and monoclinic families consists of all the reducible space group isomorphism types whose point group has infinitely many 1-dimensional invariant vector spaces. If $\Gamma$ belongs to one of these 15 isomorphism types, then $\Gamma$ has infinitely many 1-dimensional, complete, normal subgroups, and $\Gamma$ has infinitely many $2-$ dimensional, complete, normal subgroups. This is the case if and only if in the Conway et al representation of $\Gamma$ the group $\mathrm{H}$ has infinitely many proper complete normal subgroups.

The triclinic family consists of two isomorphism types (IT numbers 1 and 2). If $\Gamma$ belongs to the triclinic family, then all the geometric Seifert fibrations of $E^{3} / \Gamma$ are affinely equivalent and all the geometric co-Seifert fibrations of $E^{3} / \Gamma$ are affinely equivalent.

The monoclinic family consists of 13 isomorphism types (IT numbers 3-15). If $\Gamma$ belongs to one of these 13 isomorphism types, then $\Gamma$ has a unique 1 -dimensional, complete, characteristic subgroup $\mathrm{K}$ and a unique 2-dimensional, complete, characteristic 
subgroup $\mathrm{N}$; moreover $\mathrm{K}$ and $\mathrm{N}$ are orthogonal. The corresponding Seifert fibration is given by the primary name in Table $2 b$ of [7]. The classification of the geometric co-Seifert fibrations of $E^{3} / \Gamma$ corresponds via orthogonal duality to the Conway et al classification of the geometric Seifert fibrations of $E^{3} / \Gamma$ when $\Gamma$ belongs to the monoclinic family except for the two cases $(*: \times)$ and $(2 \mp 2: 2)$ in [7]. In Table 1, we replace the Seifert fibration $(*: \times)$ of space group IT 9 with the affine equivalent Seifert fibration $\left(*: \times_{1}\right)$, with couplings $\frac{1}{2}+\frac{1}{2}+$, and we replace the Seifert fibration $(2 \approx 2: 2)$ of space group IT 15 with the affine equivalent Seifert fibration $\left(22_{1}: 2\right)$ with couplings $0-\frac{1}{2}-\frac{1}{2}+$. With these two substitutions, indicated by a $\dagger$ in Table 1 , the classification of the geometric co-Seifert fibrations of $E^{3} / \Gamma$ under affine equivalence corresponds via orthogonal duality to the Conway et al classification of the geometric Seifert fibrations of $E^{3} / \Gamma$. See Table 1 for the orthogonal correspondence between the classifications of the Seifert and co-Seifert fibrations of $E^{3} / \Gamma$.

The first column of Table 1 is the IT number of the corresponding space group. The second column is the Conway fibrifold name of the Seifert fibration. The third column indicates whether or not the corresponding space group extension splits. If the generic fiber is a closed interval, then the space group extension splits by Theorem 18. The fourth column indicates the generic fiber of the orthogonal dual co-Seifert fibration. The fifth column indicates the base of the orthogonal dual co-Seifert fibration with a centered dot representing a circle and a dash representing a closed interval. The sixth column indicates whether or not the corresponding space group extension splits. If the generic fiber is one of the 2 -orbifolds $* 2222,22 *, 22 \times, 442, * 442,4 * 2, * 333$, $3 * 3,632, * 632$, then the space group extension splits by Theorem 18. If the base is a circle, then the space group extension splits, since $\Gamma / \mathrm{N}$ is an infinite cyclic group. The seventh column gives the index of $\mathrm{KN}$ in $\Gamma$; in particular, the index is 1 if and only if both fibrations are direct products. If the generic fiber of the Seifert fibration is a closed interval, then the base of the co-Seifert fibration is also a closed interval and the index is 1 or 2 by Theorem 20. The information in Table 1 was obtained by computer calculations. The 10 closed flat 3 -manifolds in Table 1 have IT numbers $1,4,7,9,19,29,33,76,144,169$.

Remark Our Table 1 is consistent with Table 1 in Conway et al [7], but is inconsistent with Table $2 b$ in [7] because there is a typo on page 506 of [7]. The entries $\left[\times_{0} \times{ }_{1}\right]$ and $\left[\times_{1} \times 1\right]$ of Table $2 b$ on page 506 of [7] should be switched.

Acknowledgments Finally, we wish to thank the referee for carefully reading our paper and giving us helpful comments and corrections. 
Seifert fibration co-Seifert fibration no. fibrifold split fiber base split ind.

\begin{tabular}{|c|c|c|c|c|c|c|}
\hline 1 & (o) & Yes & $\circ$ & · & Yes & 1 \\
\hline 2 & $(2222)$ & Yes & $\circ$ & - & Yes & 2 \\
\hline 3 & $\left(*_{0} *_{0}\right)$ & Yes & $\circ$ & - & Yes & 2 \\
\hline 3 & $\left(2_{0} 2_{0} 2_{0} 2_{0}\right)$ & Yes & 2222 & . & Yes & 1 \\
\hline 4 & $(\bar{x} \bar{x})$ & Yes & $\circ$ & - & No & 2 \\
\hline 4 & $\left(2{ }_{1} 2_{1} 2_{1} 2_{1}\right)$ & No & $\circ$ & . & Yes & 2 \\
\hline 5 & $(* \bar{x})$ & Yes & $\circ$ & - & Yes & 2 \\
\hline 5 & $\left(*_{1} *_{1}\right)$ & No & $\circ$ & - & No & 4 \\
\hline 5 & $\left(2_{0} 2_{0} 2_{1} 2_{1}\right)$ & No & 2222 & . & Yes & 2 \\
\hline 6 & {$\left[\circ_{0}\right]$} & Yes & $\circ$ & - & Yes & 1 \\
\hline 6 & $(* \cdot * \cdot)$ & Yes & $* *$ & . & Yes & 1 \\
\hline 7 & $\left(\overline{\mathrm{o}}_{0}\right)$ & Yes & $\circ$ & - & No & 2 \\
\hline 7 & $\left(\times x_{0}\right)$ & Yes & $x \times$ & . & Yes & 1 \\
\hline 7 & $(*: *:)$ & No & $\circ$ & . & Yes & 2 \\
\hline 8 & {$\left[o_{1}\right]$} & Yes & $\circ$ & - & No & 2 \\
\hline 8 & $(* \cdot \times)$ & Yes & $* \times$ & . & Yes & 1 \\
\hline 8 & $(* \cdot *:)$ & No & $* *$ & . & Yes & 2 \\
\hline 9 & $\left(\overline{\mathrm{o}}_{1}\right)$ & No & $\circ$ & - & No & 4 \\
\hline 9 & $\left(\times \times_{1}\right)$ & No & $x \times$ & . & Yes & 2 \\
\hline 9 & $\left(*: \times_{1}\right)^{\dagger}$ & No & $\circ$ & . & Yes & 2 \\
\hline 10 & 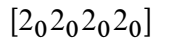 & Yes & 2222 & - & Yes & 1 \\
\hline 10 & $(* 2 \cdot 22 \cdot 2)$ & Yes & $* *$ & - & Yes & 2 \\
\hline 11 & {$\left[2_{1} 2_{1} 2_{1} 2_{1}\right]$} & Yes & $\circ$ & - & Yes & 2 \\
\hline 11 & $(22 * \cdot)$ & Yes & $* *$ & - & Yes & 2 \\
\hline 12 & {$\left[2_{0} 2_{0} 2_{1} 2_{1}\right]$} & Yes & 2222 & - & Yes & 2 \\
\hline 12 & $(2 \mp 2 \cdot 2)$ & Yes & $* \times$ & - & Yes & 2 \\
\hline 12 & $(* 2 \cdot 22: 2)$ & No & $* *$ & - & Yes & 4 \\
\hline 13 & $\left(2_{0} 2_{0} 22\right)$ & Yes & 2222 & - & Yes & 2 \\
\hline 13 & $\left(22 *_{0}\right)$ & Yes & $x \times$ & - & Yes & 2 \\
\hline 13 & $(* 2: 22: 2)$ & No & $\circ$ & - & Yes & 4 \\
\hline 14 & $\left(2{ }_{1} 2_{1} 22\right)$ & No & $\circ$ & - & No & 4 \\
\hline 14 & $(22 \times)$ & Yes & $x \times$ & - & Yes & 2 \\
\hline 14 & $(22 *:)$ & No & $\circ$ & - & No & 4 \\
\hline 15 & $\left(2_{0} 2_{1} 22\right)$ & No & 2222 & - & Yes & 4 \\
\hline 15 & $\left(22 *_{1}\right)$ & No & $x \times$ & - & Yes & 4 \\
\hline 15 & $\left(22_{1}: 2\right)^{\dagger}$ & No & $\circ$ & - & Yes & 4 \\
\hline 16 & $\left(* 2_{0} 2_{0} 2_{0} 2_{0}\right)$ & Yes & 2222 & - & Yes & 2 \\
\hline 17 & $\left(2_{0} 2_{0} *\right)$ & Yes & 2222 & - & Yes & 2 \\
\hline 17 & $\left(* 2{ }_{1} 2_{1} 2_{1} 2_{1}\right)$ & No & $\circ$ & - & Yes & 4 \\
\hline 18 & $\left(2_{0} 2_{0} \bar{x}\right)$ & Yes & 2222 & - & No & 2 \\
\hline
\end{tabular}

Seifert fibration co-Seifert fibration no. fibrifold split fiber base split ind.

\begin{tabular}{|c|c|c|c|c|c|}
\hline 18 & $\left(2_{1} 2_{1} *\right)$ & No & $\circ$ & - & No \\
\hline 19 & $\left(2_{1} 2_{1} \bar{x}\right)$ & No & $\circ$ & - & No \\
\hline 20 & $\left(2_{0} 2_{1} *\right)$ & No & 2222 & - & No \\
\hline 20 & $\left(2_{1} * 2_{1} 2_{1}\right)$ & No & $\circ$ & - & Yes \\
\hline 21 & $\left(2_{0} * 2_{0} 2_{0}\right)$ & Yes & 2222 & - & Yes \\
\hline 21 & $\left(* 2_{0} 2_{0} 2_{1} 2_{1}\right)$ & No & 2222 & - & Yes \\
\hline 22 & $\left(* 2_{0} 2_{1} 2_{0} 2_{1}\right)$ & No & 2222 & - & Yes \\
\hline 23 & $\left(2_{1} * 2_{0} 2_{0}\right)$ & No & 2222 & - & No \\
\hline 24 & $\left(2_{0} * 2_{1} 2_{1}\right)$ & No & 2222 & - & Yes \\
\hline 25 & {$\left[*_{0} \cdot *_{0} \cdot\right]$} & Yes & $* *$ & - & Yes \\
\hline 25 & $(* \cdot 2 \cdot 2 \cdot 2 \cdot 2)$ & Yes & $* 2222$ & $\cdot$ & Yes \\
\hline 26 & {$\left[\times_{0} \times_{0}\right]$} & Yes & $\times x$ & - & Yes \\
\hline 26 & $(\bar{*} \cdot \bar{*} \cdot)$ & Yes & $* *$ & - & No \\
\hline 26 & $(* \cdot 2: 2 \cdot 2: 2)$ & No & $* *$ & . & Yes \\
\hline 27 & $\left(\bar{*}_{0} \bar{*}_{0}\right)$ & Yes & $x \times$ & - & Yes \\
\hline 27 & $(*: 2: 2: 2: 2)$ & No & 2222 & $\cdot$ & Yes \\
\hline 28 & $\left(* \cdot *_{0}\right)$ & Yes & $* *$ & - & Yes \\
\hline 28 & {$\left[*_{0}: *_{0}:\right]$} & Yes & $\circ$ & - & Yes \\
\hline 28 & $\left(2_{0} 2_{0} * \cdot\right)$ & Yes & $22 *$ & $\cdot$ & Yes \\
\hline 29 & $\left(\bar{x} \times_{0}\right)$ & Yes & $x \times$ & - & No \\
\hline 29 & $(\bar{*}: \bar{*}:)$ & No & $\circ$ & - & No \\
\hline 29 & $\left(2_{1} 2_{1} *:\right)$ & No & $x \times$ & . & Yes \\
\hline 30 & $\left(*_{0} \times_{0}\right)$ & Yes & $x \times$ & - & Yes \\
\hline 30 & $\left(\bar{*}_{1} \bar{*}_{1}\right)$ & No & $\circ$ & - & No \\
\hline 30 & $\left(2_{0} 2_{0} *:\right)$ & No & 2222 & $\cdot$ & Yes \\
\hline 31 & {$\left[\times_{1} \times_{1}\right]$} & Yes & $\circ$ & - & No \\
\hline 31 & $(* \cdot \overline{\times})$ & Yes & $* *$ & - & No \\
\hline 31 & $\left(2_{1} 2_{1} * \cdot\right)$ & No & $* *$ & $\cdot$ & Yes \\
\hline 32 & $\left(*: *_{0}\right)$ & No & $\circ$ & - & No \\
\hline 32 & $\left(2_{0} 2_{0} \times_{0}\right)$ & Yes & $22 \times$ & . & Yes \\
\hline 33 & $\left(\overline{\times} \times_{1}\right)$ & No & $\circ$ & - & No \\
\hline 33 & $(*: \bar{x})$ & No & $\circ$ & - & No \\
\hline 33 & $\left(2_{1} 2_{1} \times\right)$ & No & $x \times$ & $\cdot$ & Yes \\
\hline 34 & $\left(*_{0} \times_{1}\right)$ & No & $\circ$ & - & No \\
\hline 34 & $\left(2_{0} 2_{0} \times{ }_{1}\right)$ & No & 2222 & . & Yes \\
\hline 35 & {$\left[*_{0} \cdot *_{0}:\right]$} & Yes & $* *$ & - & Yes \\
\hline 35 & $\left(2_{0} * \cdot 2 \cdot 2\right)$ & Yes & $2 * 22$ & . & Yes \\
\hline 36 & {$\left[\times_{0} \times_{1}\right]$} & Yes & $\times \times$ & - & No \\
\hline 36 & $(\bar{*} \cdot \bar{*}:)$ & No & $* *$ & - & No \\
\hline 36 & $\left(2_{1} * \cdot 2: 2\right)$ & No & $* \times$ & . & Yes \\
\hline
\end{tabular}

Table 1: Three-dimensional crystallographic Seifert and co-Seifert fibrations 
Seifert fibration co-Seifert fibration no. fibrifold split fiber base split ind.

\begin{tabular}{|c|c|c|c|c|}
\hline $37\left(\bar{*}_{0} \bar{*}_{1}\right)$ & No & $x \times$ & - & Yes \\
\hline $37\left(2_{0} *: 2: 2\right)$ & No & 2222 & & Yes \\
\hline $38\left[* \cdot \times_{0}\right]$ & Yes & $* x$ & - & Yes \\
\hline $38\left[*_{1} \cdot *_{1} \cdot\right]$ & es & & - & Jo \\
\hline $38(* \cdot 2 \cdot 2 \cdot 2: 2)$ & o & & $\cdot$ & Yes \\
\hline $39\left({\left.\bar{*} \cdot \bar{*}_{0}\right)}\right.$ & & $* \times$ & - & \\
\hline $39\left[*_{1}: *_{1}:\right]$ & & $x \times$ & - & Tes \\
\hline $39(* \cdot 2: 2: 2: 2)$ & $\mathrm{N}$ & 22 & & es \\
\hline $40\left[*: \times_{1}\right]$ & & ○ & - & ES \\
\hline $40(* \cdot * 1)$ & & $* *$ & - & Jo \\
\hline $40\left(2_{0} 2_{1} * \cdot\right)$ & & $22 x$ & . & Yes \\
\hline $41\left(\bar{*}^{\prime} \bar{*}_{1}\right)$ & o & $\circ$ & - & No \\
\hline $41 \quad\left(*: *_{1}\right)$ & & $x \times$ & - & \\
\hline $41 \quad\left(2_{0} 2_{1} *:\right)$ & & 22 & . & Tes \\
\hline $42\left[*_{1} \cdot *_{1}:\right]$ & & $* \times$ & - & \\
\hline $42(* \cdot 2 \cdot 2: 2: 2)$ & & & $\cdot$ & \\
\hline $43\left(*_{1} \times\right)$ & & $\circ$ & - & \\
\hline $43\left(2_{0} 2_{1} \times\right)$ & & & $\cdot$ & \\
\hline $44\left[* \cdot \times_{1}\right]$ & & $* *$ & - & o \\
\hline $44\left(2_{1} * \cdot 2 \cdot 2\right)$ & & & $\cdot$ & \\
\hline $45\left(\bar{*}: \bar{*}_{0}\right)$ & & $\times>$ & - & \\
\hline $45 \quad\left(2_{1} *: 2: 2\right)$ & & $22 \times$ & . & \\
\hline $46\left[*: \times_{0}\right]$ & & $x \times$ & - & \\
\hline $46\left(\bar{*}^{\prime} \bar{*}_{1}\right)$ & & $* *$ & - & \\
\hline $46\left(2_{0} * \cdot 2: 2\right.$ & & 22 & . & \\
\hline $47[* \cdot 2 \cdot 2 \cdot 2$ & & & - & Yes \\
\hline $48\left(2 \bar{*}_{1} 2_{0}\right.$ & & & - & \\
\hline $49\left(* 2_{0} 2_{0}\right.$ & Yes & $22 *$ & - & Yes \\
\hline $49[*: 2: 2: 2$ & & & - & \\
\hline $50\left(2 \bar{*}_{0} 2_{0} 2_{0}\right)$ & Y & $22>$ & - & Yes \\
\hline $50\left(* 2_{0} 2_{0} 2: 2\right)$ & & & - & \\
\hline $51\left[2_{0} 2_{0} * \cdot\right]$ & Yes & $22 *$ & - & Yes \\
\hline $51 \quad(* 2 \cdot 2 \cdot 2 \cdot 2)$ & & & - & es \\
\hline $51[* \cdot 2: 2 \cdot 2: 2]$ & $\mathrm{Y}$ & $* *$ & - & Yes \\
\hline $52\left(2_{0} 2 \bar{*}_{1}\right)$ & & 2222 & - & Yes \\
\hline $52\left(22_{2} 2_{1}\right)$ & No & $x \times$ & - & Yes \\
\hline $52\left(2_{0} * 2: 2\right)$ & & 222 & - & Yes \\
\hline $53\left[2_{0} 2_{0} *:\right]$ & Yes & 2222 & - & Yes \\
\hline $53\left(2_{0} * 2 \cdot 2\right)$ & Yes & $22 *$ & - & Yes \\
\hline $53\left(* 2_{1} 2_{1} 2 \cdot 2\right)$ & No & $* *$ & - & Yes \\
\hline
\end{tabular}

Seifert fibration co-Seifert fibration

no. fibrifold split fiber base split ind.

\begin{tabular}{|c|c|c|c|c|}
\hline $54\left(2_{0} 2 \bar{*}_{0}\right)$ & Yes & $22 x$ & - & $\mathrm{Ye}$ \\
\hline $54\left(* 2{ }_{1} 2_{1} 2: 2\right)$ & No & $x \times$ & - & Yes \\
\hline $54(* 2: 2: 2: 2)$ & No & 2222 & & Yes \\
\hline $5\left[2_{0} 2_{0} \times_{0}\right]$ & Yes & $22 \times$ & & Yes \\
\hline $5(* 2 \cdot 2: 2 \cdot 2)$ & No & $* *$ & & No \\
\hline $6\left(2{ }_{1} 2 \bar{*}_{0}\right)$ & No & $x \times$ & 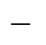 & Yes \\
\hline $6(2 \bar{*}: 2$ & No & & & No \\
\hline $7\left(2_{0} 2\right.$ & Yes & $22 *$ & . & Yes \\
\hline $57\left[2{ }_{1} 2_{1} *\right.$ & Yes & $x \times$ & - & Yes \\
\hline $7(* 2: 2$ & No & $* *$ & - & Yes \\
\hline $8\left[2_{0} 2_{0} \times{ }_{1}\right]$ & Yes & 2222 & - & No \\
\hline $8\left(2_{1} * 2 \cdot 2\right)$ & No & $* *$ & - & No \\
\hline $59\left[2_{1} 2_{1} * \cdot\right]$ & Yes & $* *$ & - & Yes \\
\hline $59(2 \bar{*} \cdot 2 \cdot 2)$ & Yes & $* 222$ & - & Yes \\
\hline $0 \quad\left(2{ }_{1} 2\right.$ & No & $x \times$ & - & No \\
\hline $60 \quad\left(2_{0} 2 \bar{*}:\right)$ & No & 2222 & - & No \\
\hline $50 \quad\left(2_{1} * 2: 2\right)$ & No & $x \times$ & - & Yes \\
\hline $61 \quad\left(2_{1} 2 \bar{*}:\right)$ & o & $x \times$ & - & No \\
\hline $62\left[2{ }_{1} 2_{1} \times\right]$ & Yes & $x \times$ & - & Yes \\
\hline $62\left(2{ }_{1} 2 \bar{*} \cdot\right)$ & No & $* *$ & - & No \\
\hline $52(2 \%$ & o & $* *$ & - & No \\
\hline $63\left[2_{0} 2_{1} * \cdot\right]$ & Yes & $22 *$ & - & Yes \\
\hline $63\left[2{ }_{1} * \cdot 2: 2\right]$ & Yes & $* \times$ & - & Yes \\
\hline $63(* 2 \cdot 2 \cdot 2: 2)$ & No & & - & Yes \\
\hline $64\left[2_{0} 2_{1} *:\right]$ & Yes & $22 x$ & - & Yes \\
\hline $64\left(* 2{ }_{1} 2 \cdot 2: 2\right)$ & No & $* \times$ & - & Yes \\
\hline $64(* 2 \cdot 2: 2: 2)$ & No & $22 *$ & - & Yes \\
\hline $65\left[2_{0} * \cdot 2 \cdot 2\right]$ & Yes & $2 *$ & - & Yes \\
\hline $65[* \cdot 2 \cdot 2 \cdot 2: 2]$ & Yes & $* 22$ & - & Yes \\
\hline $66\left[2_{0} *: 2: 2\right]$ & Yes & 222 & - & Yes \\
\hline $66\left(* 2{ }_{0} 2_{1} 2 \cdot 2\right)$ & No & $22 *$ & - & Yes \\
\hline $67\left(* 2{ }_{0} 2 \cdot 2 \cdot 2\right)$ & Yes & $2 * 22$ & - & Yes \\
\hline $67[* \cdot 2: 2: 2: 2]$ & Yes & $22 *$ & - & Yes \\
\hline $68\left(* 2{ }_{0} 2_{1} 2: 2\right)$ & No & $22 \times$ & - & Yes \\
\hline $68\left(* 2{ }_{0} 2: 2: 2\right)$ & No & 2222 & - & Yes \\
\hline $69[* \cdot 2 \cdot 2: 2: 2]$ & Yes & $2 * 22$ & - & Yes \\
\hline $70\left(2 \bar{*} 2_{0} 2_{1}\right)$ & No & 2222 & - & Yes \\
\hline $71\left[2{ }_{1} * \cdot 2 \cdot 2\right]$ & Yes & $* 2222$ & - & Yes \\
\hline $72\left[2_{1} *: 2: 2\right]$ & Yes & $22 \times$ & - & Yes \\
\hline $72\left(* 2_{0} 2 \cdot 2: 2\right)$ & No & $22 *$ & - & Yes \\
\hline
\end{tabular}

Table 1 (cont): Three-dimensional crystallographic Seifert and co-Seifert fibrations 
Seifert fibration co-Seifert fibration no. fibrifold split fiber base split ind.

\begin{tabular}{|c|c|c|c|c|c|}
\hline 73 & $\left(* 2_{1} 2: 2: 2\right)$ & No & $22 \times$ & - & Yes \\
\hline 74 & {$\left[2_{0} * \cdot 2: 2\right]$} & Yes & $22 *$ & - & Yes \\
\hline 74 & $\left(* 2_{1} 2 \cdot 2 \cdot 2\right)$ & No & $* 2222$ & - & Yes \\
\hline 5 & $\left(4_{0} 4_{0} 2_{0}\right)$ & Yes & 442 & . & Yes \\
\hline 76 & $\left(4_{1} 4_{1} 2_{1}\right)$ & No & $\circ$ & . & Yes \\
\hline 77 & $\left(4_{2} 4_{2} 2_{0}\right)$ & No & 2222 & . & Yes \\
\hline 79 & $\left(4_{2} 4_{0} 2_{1}\right)$ & No & 442 & ${ }^{\circ}$ & Yes \\
\hline 80 & $\left(4_{3} 4_{1} 2_{0}\right)$ & No & 2222 & . & Yes \\
\hline 81 & $\left(442_{0}\right)$ & Yes & & - & No \\
\hline & $\left(442_{1}\right)$ & No & 2222 & - & No \\
\hline 83 & {$\left[4_{0} 4_{0} 2_{0}\right]$} & Yes & 442 & - & Yes \\
\hline 84 & {$\left[4_{2} 4_{2} 2_{0}\right]$} & Yes & 2222 & - & No \\
\hline 85 & $\left(44_{0} 2\right)$ & Yes & 442 & - & Yes \\
\hline 86 & $\left(44_{2} 2\right)$ & No & 2222 & - & No \\
\hline 87 & {$\left[4_{2} 4_{0} 2_{1}\right]$} & Yes & & - & Yes \\
\hline 88 & $\left(44_{1} 2\right)$ & No & & - & No \\
\hline 89 & $\left(* 4_{0} 4_{0} 2_{0}\right)$ & Yes & & - & Yes \\
\hline 90 & $\left(4_{0} * 2_{0}\right)$ & Yes & 44 & - & Yes \\
\hline 91 & $\left(* 4_{1} 4_{1} 2_{1}\right)$ & No & $\circ$ & - & Yes \\
\hline 92 & $\left(4_{1} * 2_{1}\right)$ & No & $\circ$ & - & No \\
\hline 93 & $\left(* 4_{2} 4_{2} 2_{0}\right)$ & No & & - & Yes \\
\hline 94 & $\left(4_{2} * 2_{0}\right)$ & No & 222 & - & No \\
\hline 97 & $\left(* 4_{2} 4_{0} 2_{1}\right)$ & No & & - & Yes \\
\hline 98 & $\left(* 4_{3} 4_{1} 2_{0}\right)$ & No & 22 & - & Yes \\
\hline 99 & $(* \cdot 4 \cdot 4 \cdot 2)$ & Yes & & . & Yes \\
\hline 100 & $\left(4_{0} *\right.$ & Yes & 4 & . & Yes \\
\hline & $(*: 4$ & No & & . & Yes \\
\hline 102 & $\left(4_{2} * \cdot 2\right)$ & No & $2 * 22$ & . & Yes \\
\hline & & No & & . & Yes \\
\hline 104 & $\left(4_{0} *:\right.$ & No & 44 & . & Yes \\
\hline & & No & & . & Yes \\
\hline 106 & $\left(4_{2} *: 2\right)$ & No & $22 x$ & . & Yes \\
\hline 107 & $(* \cdot 4 \cdot 4: 2)$ & No & $* 442$ & . & Yes \\
\hline 108 & $(* \cdot 4: 4: 2)$ & No & $4 * 2$ & . & Yes \\
\hline & $\left(4_{1} * \cdot 2\right)$ & No & $* 2222$ & . & Yes \\
\hline & $\left(4_{1} *: 2\right)$ & No & $22 \times$ & $\cdot$ & Yes \\
\hline & $\left(* 4 \cdot 42_{0}\right)$ & Yes & & - & Yes \\
\hline & $\left(* 4: 42_{0}\right)$ & No & 2222 & - & No \\
\hline & $(4 \bar{*} \cdot 2)$ & Yes & $2 * 22$ & - & No \\
\hline 114 & $(4 \bar{*}: 2)$ & No & 2222 & - & No \\
\hline
\end{tabular}

Seifert fibration co-Seifert fibration

no. fibrifold split fiber base split ind.

\begin{tabular}{|c|c|c|c|c|}
\hline $15(* \cdot 44$ & Yes & $* 2222$ & - & \\
\hline $16(*: 44: 2)$ & No & 2222 & - & \\
\hline $17\left(4 \bar{*}_{0} 2_{0}\right)$ & & $22 \times$ & - & \\
\hline $18\left(4 \bar{*}_{1} 2_{0}\right)$ & No & & - & No \\
\hline $119\left(* 4 \cdot 42_{1}\right)$ & No & $* 2222$ & - & Yes \\
\hline $20\left(* 4: 42_{1}\right)$ & No & $22 \times$ & - & Yes \\
\hline $21 \quad(* \cdot 44$ & No & & - & No \\
\hline $22\left(4 \bar{*} 2_{1}\right)$ & o & & - & No \\
\hline $123[* \cdot 4 \cdot 4 \cdot 2]$ & Yes & & - & Yes \\
\hline $24[*: 4:$ & & 442 & - & Yes \\
\hline $125\left(* 4_{0}\right.$ & & & - & Yes \\
\hline $26\left(* 4_{0}\right.$ & No & 442 & - & Yes \\
\hline $127\left[4_{0} * \cdot\right.$ & Yes & $4 * 2$ & - & Yes \\
\hline $28\left[4_{0} *: 2\right]$ & Yes & 442 & - & Yes \\
\hline $129(* 4$ & & & - & es \\
\hline $30(* 4$ & & 44 & - & es \\
\hline $31[* \cdot 4$ & & & - & es \\
\hline $32[*: 4 \cdot$ & & $2 *$ & - & Yes \\
\hline $33\left(* 4_{2} 4\right.$ & o & $22 \times$ & - & Yes \\
\hline $34\left(* 4_{2} 4 \cdot 2\right)$ & & $2 * 22$ & - & Yes \\
\hline $35\left[4_{2} *: 2\right]$ & Yes & $22 \times$ & - & Yes \\
\hline $36\left[4_{2} * \cdot 2\right]$ & Yes & $2 * 22$ & - & No \\
\hline $37(* 4$ & No & $* 2222$ & - & Yes \\
\hline $38(* 4$ : & & $2 *$ & - & \\
\hline $139[* \cdot 4 \cdot 4$ & es & $* 442$ & - & Yes \\
\hline $140[* \cdot 4: 4$ & $\mathrm{Y}$ & & - & Yes \\
\hline $141\left(* 4_{1} 4\right.$. & No & $* 2222$ & - & Yes \\
\hline $142\left(* 4_{1} 4:\right.$ & No & $22 \times$ & - & Yes \\
\hline $143\left(3_{0} 3_{0} 3_{0}\right)$ & Yes & 333 & $\cdot$ & Yes \\
\hline $144\left(3_{1} 3_{1} 3_{1}\right)$ & No & $\circ$ & $\cdot$ & Yes \\
\hline $146\left(3_{0} 3_{1} 3_{2}\right)$ & No & 333 & $\cdot$ & Yes \\
\hline $147\left(63_{0} 2\right)$ & $\mathrm{Ye}$ & & - & Yes \\
\hline $148\left(63_{1} 2\right)$ & No & 333 & - & Yes \\
\hline $149\left(* 3_{0} 3_{0} 3_{0}\right)$ & Yes & 333 & - & Yes \\
\hline $150\left(3_{0} * 3_{0}\right)$ & Yes & 333 & - & Yes \\
\hline $151\left(* 3_{1} 3_{1} 3_{1}\right)$ & No & $\circ$ & - & Yes \\
\hline $152\left(3_{1} * 3_{1}\right)$ & No & $\circ$ & - & Yes \\
\hline $155\left(* 3_{0} 3_{1} 3_{2}\right)$ & No & 333 & - & Yes \\
\hline $156(* \cdot 3 \cdot 3 \cdot 3)$ & Yes & *333 & . & Yes \\
\hline $157\left(3_{0} * \cdot 3\right)$ & Yes & $3 * 3$ & . & Yes \\
\hline
\end{tabular}

Table 1 (cont): Three-dimensional crystallographic Seifert and co-Seifert fibrations 


\begin{tabular}{|c|c|c|c|c|c|}
\hline \multicolumn{2}{|c|}{ Seifert fibration } & \multicolumn{4}{|c|}{ co-Seifert fibration } \\
\hline no. fibrifold & split & fiber & base & split & ind. \\
\hline $158(*: 3: 3: 3)$ & No & 333 & . & Yes & 2 \\
\hline $159\left(3_{0} *: 3\right)$ & No & 333 & . & Yes & 2 \\
\hline $160\left(3_{1} * \cdot 3\right)$ & No & $* 333$ & . & Yes & 3 \\
\hline $161\left(3_{1} *: 3\right)$ & No & 333 & . & Yes & 6 \\
\hline $162\left(* \cdot 63_{0} 2\right)$ & Yes & $3 * 3$ & - & Yes & 2 \\
\hline $163\left(*: 63_{0} 2\right)$ & No & 333 & - & Yes & 4 \\
\hline $164(* 6 \cdot 3 \cdot 2)$ & Yes & $* 333$ & - & Yes & 2 \\
\hline $165(* 6: 3: 2)$ & No & 333 & - & Yes & 4 \\
\hline $166\left(* \cdot 63_{1} 2\right)$ & No & $* 333$ & - & Yes & 6 \\
\hline $167\left(*: 63_{1} 2\right)$ & No & 333 & - & Yes & 12 \\
\hline $168\left(6_{0} 3_{0} 2_{0}\right)$ & Yes & 632 & . & Yes & 1 \\
\hline $169\left(6_{1} 3_{1} 2_{1}\right)$ & No & $\circ$ & . & Yes & 6 \\
\hline $171\left(6_{2} 3_{2} 2_{0}\right)$ & No & 2222 & . & Yes & 3 \\
\hline $173\left(6_{3} 3_{0} 2_{1}\right)$ & No & 333 & . & Yes & 2 \\
\hline $174\left[3_{0} 3_{0} 3_{0}\right]$ & Yes & 333 & - & Yes & 1 \\
\hline $175\left[6_{0} 3_{0} 2_{0}\right]$ & Yes & 632 & - & Yes & 1 \\
\hline $176\left[6_{3} 3_{0} 2_{1}\right]$ & Yes & 333 & - & Yes & 2 \\
\hline
\end{tabular}

\begin{tabular}{|c|c|c|c|c|c|}
\hline \multicolumn{2}{|c|}{$\underline{\text { Seifert fibration }}$} & \multicolumn{4}{|c|}{ co-Seifert fibration } \\
\hline 10. fibrifold & split & fiber & base & & ind \\
\hline $177\left(* 6_{0} 3_{0} 2_{0}\right)$ & Yes & 632 & - & Yes & 2 \\
\hline $178(* 6$ & No & $\circ$ & & Yes & 12 \\
\hline $80\left(* 6_{2} 3_{2} 2_{0}\right)$ & No & 2222 & - & & \\
\hline $82(* 63$ & No & 33 & - & Yes & 4 \\
\hline $183(* \cdot$ & Yes & & $\cdot$ & & 1 \\
\hline $34(*$ : & No & & & & 2 \\
\hline $185(*$ & No & & · & 10 & 2 \\
\hline $86(*:$ & No & & $\cdot$ & & 2 \\
\hline $87[* \cdot$ & Yes & & - & Yes & 1 \\
\hline $188[*: 3$ & Yes & & - & Yes & 2 \\
\hline $189\left[3_{0} * \cdot 3\right]$ & Yes & $3 *$ & - & Yes & 1 \\
\hline $190\left[3_{0} *: 3\right]$ & Yes & 33 & - & Yes & 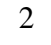 \\
\hline $191[* \cdot 6 \cdot 3$ & Yes & $* 6$ & - & Yes & 1 \\
\hline $192[*: 6: 3$ & Yes & 632 & - & Yes & \\
\hline $193[* \cdot 6: 3: 2]$ & Yes & $3 * 3$ & - & Yes & 2 \\
\hline $194[*: 6 \cdot 3 \cdot 2]$ & Yes & *333 & - & Yes & \\
\hline
\end{tabular}

Table 1 (cont): Three-dimensional crystallographic Seifert and co-Seifert fibrations

\section{References}

[1] M Boileau, S Maillot, J Porti, Three-dimensional orbifolds and their geometric structures, Panoramas et Synthèses 15, Soc. Math. France, Paris (2003) MR2060653

[2] F Bonahon, L Siebenmann, The classification of Seifert fibred 3-orbifolds, from: "Low-dimensional topology (Chelwood Gate, 1982)", (R Fenn, editor), London Math. Soc. Lecture Note Ser. 95, Cambridge Univ. Press (1985) 19-85 MR827297

[3] H Brown, R Bülow, J Neubüser, H Wondratschek, H Zassenhaus, Crystallographic groups of four-dimensional space, Wiley Monogr. in Crystallography, WileyInterscience, New York (1978) MR0484179

[4] H Brown, J Neubüser, H Zassenhaus, On integral groups. I. The reducible case, Numer. Math. 19 (1972) 386-399 MR0318334

[5] P E Conner, F Raymond, Injective operations of the toral groups, Topology 10 (1971) 283-296 MR0281218

[6] J H Conway, The orbifold notation for surface groups, from: "Groups, combinatorics \& geometry (Durham, 1990)", (M Liebeck, J Saxl, editors), London Math. Soc. Lecture Note Ser. 165, Cambridge Univ. Press (1992) 438-447 MR1200280

[7] J H Conway, O Delgado Friedrichs, DH Huson, WP Thurston, On threedimensional space groups, Beiträge Algebra Geom. 42 (2001) 475-507 MR1865535 
[8] W D Dunbar, Geometric orbifolds, Rev. Mat. Univ. Complut. Madrid 1 (1988) 67-99 MR977042

[9] D R Farkas, Crystallographic groups and their mathematics, Rocky Mountain J. Math. 11 (1981) 511-551 MR639438

[10] T Hahn (editor), International tables for crystallography. Vol. A. Space-group symmetry, second edition, D. Reidel Publishing Co., Dordrecht (1987) MR942719

[11] K B Lee, F Raymond, The role of Seifert fiber spaces in transformation groups, from: "Group actions on manifolds (Boulder, Colo., 1983)", (R Schultz, editor), Contemp. Math. 36, Amer. Math. Soc. (1985) 367-425 MR780974

[12] S Mac Lane, Homology, Classics in Math., Springer, Berlin (1995) MR1344215

[13] W Plesken, T Schulz, Counting crystallographic groups in low dimensions, Experiment. Math. 9 (2000) 407-411 MR1795312

[14] J G Ratcliffe, Foundations of hyperbolic manifolds, second edition, Graduate Texts in Math. 149, Springer, New York (2006) MR2249478

[15] J G Ratcliffe, S T Tschantz, Abelianization of space groups, Acta Crystallogr. Sect. A 65 (2009) 18-27 MR2508391

[16] D Schattschneider, The plane symmetry groups: their recognition and notation, Amer. Math. Monthly 85 (1978) 439-450 MR0477980

Department of Mathematics, Vanderbilt University

Nashville TN 37240

j.g.ratcliffe@vanderbilt.edu, steven.tschantz@vanderbilt.edu

Received: 7 January $2010 \quad$ Revised: 3 May 2010 